\title{
GEOMETRIC LOCATION OF PERIODIC POINTS OF 2-RAMIFIED POWER SERIES
}

\author{
KARL-OLOF LINDAHL AND JONAS NORDQVIST
}

\begin{abstract}
In this paper we study the geometric location of periodic points of power series defined over fields of positive characteristic $p$. We find a lower bound for the norm of all nonzero periodic points in the open unit disk of 2-ramified power series. We prove that this bound is optimal for a large class of power series. Our main technical result is a computation of the first significant terms of $p$-power iterates of 2-ramified power series. As a by-product we obtain a self-contained proof of the characterization of 2-ramified power series.
\end{abstract}

Keywords: Non-Archimedean dynamical system, difference equation, periodic point, ramification number, arithmetic dynamics

Mathematics Subject Classification: 37P05, 39A05, 11S15, $11 \mathrm{~S} 82$

\section{INTRODUCTION}

The study of periodic points is a central issue in the theory of dynamical systems. In this article we are interested in the geometric location of periodic points of power series defined over fields of positive characteristic. Dynamics over fields of positive characteristic is an important topic in arithmetic dynamics [Sil07, AK09]. Lindahl and Rivera-Letelier [Lin13, LRL16a, LRL16b] showed that there is a connection between the geometric location of periodic points of power series with integer coefficients, and lower ramification numbers of wildly ramified field automorphisms. We utilize this connection to obtain an optimal lower bound for norms of periodic points of power series having a certain sequence of lower ramification numbers.

Throughout let $p$ be a prime and $k$ be a field of characteristic $p$. Denote by $\operatorname{ord}(\cdot)$ the valuation on $k[[\zeta]]$ defined for a nonzero power series as the lowest degree of its nonzero terms, and put ord $(0)=+\infty$. Let $g \in k[[\zeta]]$ be a power series satisfying $g(0)=0$ and $g^{\prime}(0)=1$. Then for all integers $n \geq 0$ we define the corresponding lower ramification number of $g$ as

$$
i_{n}(g):=\operatorname{ord}\left(\frac{g^{p^{n}}(\zeta)-\zeta}{\zeta}\right) .
$$

A famous theorem by Sen [Sen69,Lub95,LRL16a] states that for an integer $n \geq 1$ if $i_{n}(g)<+\infty$, then $i_{n}(g) \equiv i_{n-1}(g)\left(\bmod p^{n}\right)$. Thus given $i_{0}(g) \geq 1$ we have

$$
i_{n}(g) \geq 1+p+\cdots+p^{n},
$$

for all $n \geq 1$. If (1.1) holds with equality we say that $g$ is minimally ramified. Moreover, let $b \geq 1$ be an integer, and suppose that for all integers $n \geq 0$ we have

$$
i_{n}(g)=b\left(1+p+\cdots+p^{n}\right) .
$$

Then we say that $g$ is $b$-ramified.

Of particular interest in this paper are periodic points of power series that are 2-ramified. Recall that the minimal period of each periodic point $\zeta_{0} \neq 0$ of $g$ in the open unit disk of $k$ is of the form $p^{n}$, for some integer $n \geq 0$, see for example [LRL16b, Lemma 2.1]. 
1.1. Main results. The main result of this paper is the lower bound for the norm of periodic points of 2-ramified power series given in Theorem A below. We also give sufficient conditions for optimality in this lower bound as discussed in §1.1.1.

Theorem A. Let $p \geq 5$ be a prime and let $k:=(k,|\cdot|)$ be an ultrametric field of characteristic $p$. Let $f$ be a power series with coefficients in the closed unit disk of $k$, of the form

$$
f(\zeta)=\zeta\left(1+\sum_{j=2}^{+\infty} a_{j} \zeta^{j}\right) .
$$

Put $\lambda:=a_{2}^{p-3}\left(3 / 2 a_{2}^{3}+a_{3}^{2}-a_{2} a_{4}\right)$. Let $\zeta_{0}$ be a periodic point of $f$ in the open unit disk of $k$. Then

$$
\left|\zeta_{0}\right| \geq|\lambda|^{\frac{1}{p}}
$$

provided that $\zeta_{0}$ is not a fixed point. If $\zeta_{0} \neq 0$ is a fixed point of $f$ we have $\left|\zeta_{0}\right| \geq\left|a_{2}\right|$.

Remark 1. We note that $\lambda \neq 0$ is equivalent to $f$ being 2-ramified.

Theorem A is a consequence of Theorem B and [LRL16a, Lemma 2.4].

Theorem B. Let $p$ be an odd prime and let $k$ be a field of characteristic $p$. Let $f \in k[[\zeta]]$ be defined as

$$
f(\zeta)=\zeta\left(1+\sum_{j=2}^{+\infty} a_{j} \zeta^{j}\right) .
$$

Furthermore, let $n \geq 1$ be an integer, and let

$$
d(n)=d:=\frac{p^{n}-1}{p-1}, \quad \varphi:=3 / 2 a_{2}^{3}+a_{3}^{2}-a_{2} a_{4} .
$$

Let $\alpha_{n}, \beta_{n}$ and $\gamma_{n}$ be defined as follows

Then

$$
\alpha_{n}=a_{2}^{p^{n}-2 d} \varphi^{d}, \quad \beta_{n}=\frac{a_{3}}{a_{2}} \alpha_{n}, \quad \gamma_{n}=-\left(\frac{3 a_{2}}{2}-\frac{a_{4}}{a_{2}}\right) \alpha_{n} .
$$

$$
f^{p^{n}}(\zeta)-\zeta \equiv \alpha_{n} \zeta^{2 d+1}+\beta_{n} \zeta^{2 d+2}+\gamma_{n} \zeta^{2 d+3} \bmod \left\langle\zeta^{2 d+4}\right\rangle
$$

The main work of the proof is the Main Lemma given in $\S 4$ where we compute the first significant terms of $f$ at its $p$ th iterate by solving systems of difference equations in characteristic $p$. This result is an extension of Proposition 1 in [Nor17]. We also note that due to Theorem B we have a self-contained proof of Theorem 1 in [Nor17].

Below we discuss sufficient conditions for optimality of the bound in Theorem A in terms of the reduction of $f$.

1.1.1. Optimality condition. Throughout the paper let $(k,|\cdot|)$ be an ultrametric field, $\mathcal{O}_{k}$ denote the ring of integers of $k$, and $\mathfrak{m}_{k}$ its maximal ideal. Geometrically, $\mathfrak{m}_{k}$ is the open unit disk in $k$. Let $\widetilde{k}:=\mathcal{O}_{k} / \mathfrak{m}_{k}$ be the residue field of $k$. Denote the projection in $\widetilde{k}$ of an element $a$ of $\mathcal{O}_{k}$ by $\widetilde{a}$; it is the reduction of $a$. The reduction of a power series $g \in \mathcal{O}_{k}[[\zeta]]$ is the power series $\widetilde{g}(\zeta) \in \widetilde{k}[[\zeta]]$ whose coefficients are the reductions of the corresponding coefficients of $g$.

The following result gives sufficient conditions for optimality of the bound in Theorem A.

Corollary A. Let $p \geq 5$ be a prime and let $k:=(k,|\cdot|)$ be an ultrametric field of characteristic p. Let $f \in \mathcal{O}_{k}[[\zeta]]$ be of the form

$$
f(\zeta)=\zeta\left(1+\sum_{j=2}^{+\infty} a_{j} \zeta^{j}\right)
$$


Put $\lambda:=a_{2}^{p-3}\left(3 / 2 a_{2}^{3}+a_{3}^{2}-a_{2} a_{4}\right)$. Furthermore let $\lambda \neq 0$, and $\widetilde{f}(\zeta)$ be 3-ramified. Then all periodic points of $f$ in the open unit disk of $k$ that are not fixed points, are on the sphere

$$
\left\{\zeta \in k:|\zeta|=|\lambda|^{\frac{1}{p}}\right\}
$$

Remark 2. By [LS98, Corollary 1] $\widetilde{f}(\zeta)$ is 3-ramified if $i_{0}(\widetilde{f})=3$ and $i_{1}(\widetilde{f})=3+3 p$. In particular, if $f \in k[[\zeta]]$ is of the form

$$
f(\zeta) \equiv \zeta\left(1+a_{2} \zeta^{2}+a_{3} \zeta^{3}\right) \quad \bmod \left\langle\zeta^{10}\right\rangle, 0<\left|a_{2}\right|<1 \text { and }\left|a_{3}\right|=1 .
$$

Then $\lambda \neq 0$ and $\widetilde{f}(\zeta)$ is 3-ramified. See Appendix A for details.

The following example illustrates that the condition in Corollary A that $\widetilde{f}(\zeta)$ is 3-ramified is not redundant. See Appendix B for details.

Example 1. Let $p=5, k=\mathbb{F}_{5}((t))$ and $q(\zeta) \in \mathcal{O}_{k}[[\zeta]]$ be a power series of the form

$$
q(\zeta) \equiv \zeta\left(1+c_{2} \zeta^{2}+c_{3} \zeta^{3}+c_{4} \zeta^{4}\right) \quad \bmod \left\langle\zeta^{6}\right\rangle
$$

Put $\lambda:=c_{2}^{p-3}\left(3 / 2 c_{2}^{3}+c_{3}^{2}-c_{2} c_{4}\right)$. If $\left(c_{2}, c_{3}, c_{4}\right)=(1+t, 1,0)$ then all periodic points $\zeta_{0}$ in $\mathfrak{m}_{k}$ of $g$, of minimal period $p^{n}$ with $n \geq 1$, have norm $\left|\zeta_{0}\right|=|\lambda|^{\frac{1}{p}}$. If $\left(c_{2}, c_{3}, c_{4}\right)=(2+t, 4,4)$, then $\left|\zeta_{0}\right|>|\lambda|^{\frac{1}{p}}$. In both cases $q$ is 2-ramified, but in none of the cases $\widetilde{q}$ is 3-ramified.

1.2. Related works. In [LRL16a] the authors give a corresponding result of Theorem A for minimally ramified power series, where $\lambda$ is expressed in terms of the coefficients of the first two non-linear terms. Provided the information from Theorem B we can make a corresponding version of Corollary A for minimally ramified power series, where the conditions for optimality are expressed in terms of the four lowest degree non-linear terms.

Let $\eta \in k$ and $f$ be a power series in $k[[\zeta]]$ of the form

$$
f(\zeta)=\eta \zeta+\cdots .
$$

In this paper we study the parabolic case where $\eta=1$. The irrationally indifferent case, where the multiplier $\eta$ is of norm one but not a root of unity, was studied in [LRL16b]. The $p$-adic case was also studied in [AV94, Lin13, Lub94, RL03]. In contrast to our case where the periodic points of period greater than one are concentrated on a single sphere inside the open unit disk, in the latter cases the periodic points are distributed on infinitely many different spheres.

The method used in this paper to calculate the coefficients of $p$-power iterates boils down to solving systems of difference equations over non-Archimedean fields. See for example [MA15] for a recent contribution to this field of research.

1.3. Organization of the paper. Theorem A and Corollary A is proven in section $\S 2$ assuming Theorem B. The main technical result of this paper is the computation of the first significant terms of $f$ at its $p$ th iterate which is done in the Main Lemma in $\S 4$. This result is an extension of Proposition 1 in [Nor17]. The setup for this proof is discussed in $\S 3$, and we state and prove the Main Lemma in $\S 4$ together with the proof of Theorem B.

\section{Proof of Theorem A and Corollary A assuming Theorem B}

In this section we prove Theorem A assuming Theorem B. The proof is a direct consequence of Theorem B and [LRL16a, Lemma 2.4]. Before stating the special case of [LRL16a, Lemma 2.4] utilized in the proof of Theorem A we give the following definitions.

Definition 1. Let $p$ be a prime number and $k$ field of characteristic $p$. For a power series $g(\zeta)$ in $k[[\zeta]]$ of the form

$$
g(\zeta)=\zeta+\ldots,
$$


define for each integer $n \geq 0$ the element $\mu_{n}(g)$ of $k$ as follows: Put $\mu_{n}(g):=0$ if $i_{n}(g)=+\infty$, and otherwise let $\mu_{n}(g)$ be the coefficient of $\zeta^{i_{n}(g)+1}$ in the power series $g^{p^{n}}(\zeta)-\zeta$.

For a power series $f(\zeta)$ in $\mathcal{O}_{k}[[\zeta]]$, the Weierstrass degree wideg $(f)$ of $f$ is the order in $\widetilde{k}[[z]]$ of the reduction $\widetilde{f}(z)$ of $f(\zeta)$. Note that if wideg $(f)$ is finite, the number of zeros of $f$ in $\mathfrak{m}_{k}$, counted with multiplicity, is less than or equal to wideg $(f)$; see e.g. [Lan02, §VI, Theorem 9.2].

Lemma 1 (Special case of Lemma 2.4 in [LRL16a]). Let $p$ be a prime and $(k,|\cdot|)$ an ultrametric field of characteristic $p$. Moreover, let $g(\zeta)$ be a parabolic power series in $\mathcal{O}_{k}[[\zeta]]$. Then the following properties hold.

(1) Let $w_{0} \neq 0$ in $\mathfrak{m}_{k}$ be a fixed point of $g$. Then we have

(2) Let $n \geq 1$ be an integer and $\zeta_{0}$ in $\mathfrak{m}_{k}$ a periodic point of $g$ of minimal period $p^{n}$. If in addition $i_{n}(g)<+\infty$, then we have

$$
\left|\zeta_{0}\right| \geq\left|\frac{\mu_{n}(g)}{\mu_{n-1}(g)}\right|^{\frac{1}{p^{n}}}
$$

with equality if and only if

$$
\text { wideg }\left(\frac{g^{p^{n}}(\zeta)-\zeta}{g^{p^{n-1}}(\zeta)-\zeta}\right)=i_{n}(g)-i_{n-1}(g)+p^{n} .
$$

Moreover, if (2.4) holds, then the cycle containing $\zeta_{0}$ is the only cycle of minimal period $p^{n}$ of $g$ in $\mathfrak{m}_{k}$, and for every point $\zeta_{0}^{\prime}$ in this cycle $\left|\zeta_{0}^{\prime}\right|=\left|\frac{\mu_{n}(g)}{\mu_{n-1}(g)}\right|^{\frac{1}{p^{n}}}$.

Assuming Theorem B we now have the results needed to prove Theorem A.

Proof of Theorem A. If $\lambda=0$ the theorem holds trivially. However, if $\lambda \neq 0$ then $f$ is 2ramified by Theorem B, and thus for integers $n \geq 0$ we have $\mu_{n}(f)=a_{2}^{p^{n}-2 \frac{p^{n}-1}{p-1}} \varphi^{\frac{p^{n}-1}{p-1}}$. Hence, by Lemma 1 we have for all $n \geq 1$ and all periodic points $\zeta_{0}$ in $\mathfrak{m}_{k}$ of minimal period $p^{n}$

$$
\begin{aligned}
\left|\zeta_{0}\right| \geq\left|\frac{\mu_{n}(f)}{\mu_{n-1}(f)}\right|^{\frac{1}{p^{n}}} & =\left|\frac{a_{2}^{p^{n}-2 \frac{p^{n}-1}{p-1}} \varphi^{\frac{p^{n}-1}{p-1}}}{a_{2}^{p^{n-1}-2 \frac{p^{n-1}-1}{p-1}} \varphi^{\frac{p^{n-1}-1}{p-1}}}\right|^{\frac{1}{p^{n}}} \\
& =\left|a_{2}^{(p-3) p^{n-1}} \varphi^{p^{n-1}}\right|^{\frac{1}{p^{n}}} \\
& =\left|a_{2}^{p-3} \varphi\right|^{\frac{1}{p}}=|\lambda|^{\frac{1}{p}}
\end{aligned}
$$

It follows immediately from Lemma 1 that for fixed points $\zeta_{0} \neq 0$ of $f$ in the open unit disk of $k$ we have $\left|\zeta_{0}\right| \geq\left|a_{2}\right|$. This completes the proof of Theorem A.

Proof of Corollary A. We note that $\lambda \neq 0$ implies that $f$ is 2-ramified and this in turn implies that for integers $n \geq 1$ we have $i_{n}(f)-i_{n-1}(f)+p^{n}=3 p^{n}$. Also, if $\widetilde{f}(\zeta)$ is 3-ramified then for 
$n \geq 1$ we have

$$
\begin{aligned}
\text { wideg }\left(\frac{f^{p^{n}}(\zeta)-\zeta}{f^{p^{n-1}}(\zeta)-\zeta}\right) & =\operatorname{wideg}\left(f^{p^{n}}(\zeta)-\zeta\right)-\operatorname{wideg}\left(f^{p^{n-1}}(\zeta)-\zeta\right) \\
& =3 \frac{p^{n}-1}{p-1}-3 \frac{p^{n-1}-1}{p-1}=3 p^{n}
\end{aligned}
$$

Hence, (2.4) in Lemma 1 holds with equality. This completes the proof of Corollary A.

\section{TeChnical Results}

In this section we present results that we use to prove our Main Lemma and Theorem B.

Throughout the paper for any nonnegative integer $n$ let $n$ !! denote the double factorial of $n$. We put $0 ! !:=1$ and $1 ! !:=1$. For future reference, we note that for integers $n \geq 2$, we have

$$
n ! !=n(n-2) ! !
$$

The proof of the Main Lemma relies on solving linear difference equations expressed as sums of rational functions. For convenience these sums are considered over the $p$-adic numbers $\mathbb{Q}_{p}$ and its ring of integers $\mathbb{Z}_{p}$. The main part of this section involves finding the corresponding reductions in $\mathbb{F}_{p}$. Throughout we let $\nu_{p}(\cdot)$ denote the $p$-adic valuation.

Definition 2. Let $p$ be a prime, and let $f: \mathbb{Q}_{p} \rightarrow \mathbb{Q}_{p}$. We say that $f$ has a pole at a if $f(a) \notin \mathbb{Z}_{p}$. Furthermore, if $p^{n} f(a) \in \mathbb{Z}_{p}$ but $p^{n-1} f(a) \notin \mathbb{Z}_{p}$, then we say that the pole is of order $n$. A pole of order 1 is called a simple pole. Moreover, we define the residue of a function at a pole a of order $n$ as $p^{n} f(a)$.

Lemma 2. Let $p$ be a prime and let $f: \mathbb{Q}_{p} \rightarrow \mathbb{Q}_{p}$ be such that $f$ only has simple poles. Put

$$
F:=p \sum_{j=n}^{N} f(j)
$$

Then the reduction $\widetilde{F}$ is well-defined and

$$
\widetilde{F}=\sum_{\substack{n \leq a \leq N \\ a \text { is a pole of } f}} p f(a) .
$$

Proof. Using that $f$ only has simple poles we see that the reduction of $p f(a)$ is well-defined, and the proof of the lemma follows from seeing that for all elements $b \in[n, N]$ such that $b$ is not a pole of $f$ we have $p f(b) \equiv 0 \bmod p \mathbb{Z}_{p}$.

Lemma 3. Let $p$ be a prime, $a, b \in \mathbb{F}_{p}$, and $a \neq 0$. Furthermore let $f(n)=$ an $+b, s^{\prime} \equiv-a^{-1} b$ $(\bmod p)$ and $S=\mathbb{F}_{p} \backslash\left\{s^{\prime}\right\}$. Then

$$
\prod_{s \in S} f(s) \equiv-1 \quad(\bmod p) .
$$

Proof. This is a consequence of Wilson's theorem, and the fact that any linear function defined on $\mathbb{F}_{p}$ simply permutes the elements of $\mathbb{F}_{p}$.

The following two lemmas are slightly reformulated versions of Lemma 2 and 3 in [Nor17].

Lemma 4. Let $p$ be an odd prime. For each integer $n \geq 1$ let $\mathcal{R}_{n}$ and $\mathcal{T}_{n}$ in $\mathbb{Q}_{p}$ be defined by

$$
\mathcal{R}_{n}:=(2 n-1) ! ! \sum_{r=1}^{n}\left[\prod_{j=r+1}^{n} \frac{2 j}{2 j-1}\right]
$$


and

$$
\mathcal{T}_{n}:=(2 n+1) ! ! \sum_{j=1}^{n} \frac{(2 j) ! !}{(2 j+1) ! !}
$$

Then

$$
\mathcal{R}_{n}=(2 n+1) ! !-(2 n) ! ! \text { and } \mathcal{T}_{n}=(2 n+2) ! !-2(2 n+1) ! !
$$

In particular, $\widetilde{\mathcal{R}}_{p}=\widetilde{\mathcal{T}}_{p}=0, \widetilde{\mathcal{R}}_{p-1}=1, \widetilde{\mathcal{T}}_{p-1}=0$ and $\widetilde{\mathcal{R}}_{p-2}=-1 / 2, \widetilde{\mathcal{T}}_{p-2}=-1$.

Proof. All parts of the proof except for the evaluation of $\widetilde{\mathcal{R}}_{n}$ and $\widetilde{\mathcal{T}}_{n}$ at $n=p-1$ and $n=p-2$ are given in [Nor17]. Thus to complete the proof we compute $\widetilde{\mathcal{R}}_{p-1}, \widetilde{\mathcal{R}}_{p-2}, \widetilde{\mathcal{T}}_{p-1}$ and $\widetilde{\mathcal{T}}_{p-2}$. We note that $(2 n) ! !=2^{n} n !$, and that $\nu_{p}((2 p-3) ! !)=1$ and thus we have $\widetilde{\mathcal{R}}_{p-1}=0-2^{p-1}(p-1) !=$ $0-(-1)$, and $\widetilde{\mathcal{R}}_{p-2}=0-2^{p-2}(p-2) !=-1 / 2$. Also note that $\nu_{p}((2 p) ! !)=\nu_{p}((2 p-1) ! !)=1$. Consequently $\widetilde{\mathcal{T}}_{p-1}=0$, and $\widetilde{\mathcal{T}}_{p-2}=(2 p-2) ! !-0=-1$.

Lemma 5. Let $p$ be an odd prime and let $a, b$ be integers. For every integer $n \geq 1$ let $\mathcal{S}_{n}(a, b)$ in $\mathbb{Q}_{p}$ be defined by

$$
\mathcal{S}_{n}(a, b):=(2 n+1) ! ! \sum_{j=1}^{n} \frac{a j+b}{2 j+1}
$$

Then $\mathcal{S}_{n}(a, b) \in \mathbb{Z}_{p}$ and in particular $\widetilde{\mathcal{S}}_{p}(a, b)=\widetilde{\mathcal{S}}_{p-1}(a, b)=a / 2-b$, and $\widetilde{\mathcal{S}}_{p-2}=-a / 2+b$.

Proof. As for the proof of Lemma 4 the proof is given in [Nor17] except for the calculations of $\widetilde{\mathcal{S}}_{p-1}$ and $\widetilde{\mathcal{S}}_{p-2}$. Note that for $j$ in $[1, p-1]$ the function $f(j)=(a j+b) /(2 j+1)$ has exactly one pole. This pole occurs for $j=(p-1) / 2$ and is of order one. Moreover, by Lemma $3, \frac{(2 p-1) ! !}{p}=-1(\bmod p)$. Consequently by Lemma $2 \widetilde{\mathcal{S}}_{p-1}=-\left(\frac{a(p-1)}{2}+b\right)$, and $\widetilde{\mathcal{S}}_{p-2}=\left(\frac{a(p-1)}{2}+b\right)$.

The main idea behind finding the reductions of the sums of rational functions we set out to find, is to compute and sum over its residues. In Lemma 6,7 and 8 we discuss three different types of functions that will repeatedly occur in later lemmas, and we address how to compute their corresponding reductions, or in the case of Lemma 8, how to express it in simpler terms.

Lemma 6. Let $p$ be an odd prime. Let $f: \mathbb{Z}_{p} \rightarrow \mathbb{Z}_{p}$, and for each integer $n \geq 1$ let $\mathcal{F}_{n} \in \mathbb{Q}_{p}$ be defined as

$$
\mathcal{F}_{n}=(2 n+2) ! ! \sum_{j=1}^{n-1} \frac{f(j)}{(2 j+4) ! !}
$$

Then $\mathcal{F}_{n} \in \mathbb{Z}_{p}$ and

$$
\widetilde{\mathcal{F}}_{p}=2 \tilde{f}(p-2)+\widetilde{f}(p-1)
$$

Lemma 7. Let $p \geq 7$ be a prime. Let $q \in \mathbb{Z}_{p}[x]$ be a polynomial such that $\operatorname{deg}(q)<(p+3) / 2$, and for each integer $n \geq 1$ let $\mathcal{F}_{n}^{\prime} \in \mathbb{Q}_{p}$ be defined as

$$
\mathcal{F}_{n}^{\prime}:=(2 n+3) ! ! \sum_{j=1}^{n-1} \frac{q(j)(2 j) ! !}{(2 j+5) ! !} .
$$

Then $\mathcal{F}_{n}^{\prime} \in \mathbb{Z}_{p}$ and $\widetilde{\mathcal{F}}_{p}^{\prime}=0$. 
Lemma 8. Let $p$ be an odd prime and let $f: \mathbb{Q}_{p} \rightarrow \mathbb{Q}_{p}$. Furthermore, for any integer $n \geq 1$ let $\mathcal{F}_{n}^{\prime \prime}$ in $\mathbb{Q}_{p}$ be defined as

$$
\mathcal{F}_{n}^{\prime \prime}:=(2 n+3) ! ! \sum_{j=1}^{n-1} \frac{(2 j+4) ! !}{(2 j+5) ! !} \sum_{i=1}^{j-1} f(i) .
$$

Then

$$
\mathcal{F}_{n}^{\prime \prime}=(2 n+4) ! ! \sum_{i=1}^{n-1} f(i)-(2 n+3) ! ! \sum_{i=1}^{n-1} f(i) \frac{(2 i+6) ! !}{(2 i+5) ! !} .
$$

Proof of Lemma 6. The lemma is a direct consequence of the fact that by Lemma 2

$$
\mathcal{F}_{p} \equiv(2 p+2) ! !\left(\frac{f(p-2)}{(2 p) ! !}+\frac{f(p-1)}{(2 p+2) ! !}\right) \quad \bmod p \mathbb{Z}_{p} .
$$

The proof of Lemma 7 and Lemma 8 follows after the statement and proof of the following lemma, which will be utilized in the proof of Lemma 7 .

Lemma 9. Let $p \geq 7$ be a prime. Furthermore, let $n \geq 2$ and $k \geq 0$ be integers, and define $\mathcal{K}(n, k)$ in $\mathbb{Q}_{p}$ as

$$
\mathcal{K}(n, k):=\sum_{j=1}^{n-1} \frac{(2(j+k)) ! !}{(2 j+5) ! !}
$$

Then

$$
\mathcal{K}(n, k)=\frac{(2(n+k)) ! !}{(2 k-3)(2 n+3) ! !}-\frac{(2 k+2) ! !}{(2 k-3) 5 ! !} .
$$

In particular for $k<(p+3) / 2$, we have

$$
\mathcal{K}(p, k) \in \mathbb{Z}_{p} .
$$

Proof. We proceed by induction in $n$. Note that for $n=2$, by definition

$$
\mathcal{K}(2, k)=\sum_{j=1}^{1} \frac{(2(j+k)) ! !}{(2 j+5) ! !}=\frac{(2 k+2) ! !}{7 ! !} .
$$

On the other hand

$$
\frac{(2(2+k)) ! !}{(2 k-3) 7 ! !}-\frac{(2 k+2) ! !}{(2 k-3) 5 ! !}=\frac{(2 k+2) ! !}{(2 k-3) 5 ! !}\left(\frac{(2 k+4)}{7}-1\right)=\frac{(2 k+2) ! !}{7 ! !} .
$$

This proves (3.4) for $n=2$. Assume that (3.4) is valid for $n \geq 2$. Then

$$
\begin{aligned}
\mathcal{K}(n+1, k) & =\sum_{j=1}^{n} \frac{(2(j+k)) ! !}{(2 j+5) ! !} \\
& =\frac{(2(n+k)) ! !}{(2 n+5) ! !}+\sum_{j=1}^{n-1} \frac{(2(j+k)) ! !}{(2 j+5) ! !} .
\end{aligned}
$$


By the induction hypothesis we obtain

$$
\begin{aligned}
\mathcal{K}(n+1, k) & =\frac{(2(n+k)) ! !}{(2 n+5) ! !}+\frac{(2(n+k)) ! !}{(2 k-3)(2 n+3) ! !}-\frac{(2 k+2) ! !}{(2 k-3) 5 ! !} \\
& =\frac{(2(n+k)) ! !(2 k-3)+(2(n+k)) ! !(2 n+5)}{(2 k-3)(2 n+5) ! !}-\frac{(2 k+2) ! !}{(2 k-3) 5 ! !} \\
& =\frac{(2(n+k)) ! !(2 k-3+2 n+5)}{(2 k-3)(2 n+5) ! !}-\frac{(2 k+2) ! !}{(2 k-3) 5 ! !} \\
& =\frac{(2(n+k)+2) ! !}{(2 k-3)(2 n+5) ! !}-\frac{(2 k+2) ! !}{(2 k-3) 5 ! !}
\end{aligned}
$$

which completes the induction step. The second statement of the lemma follows by letting $n=p$ in (3.4). Clearly $\nu_{p}((2 p+2 k) ! !)=\nu_{p}((2 p+5) ! !)=1$. Moreover for $k<(p+3) / 2$ we have $\nu_{p}(2 k-3)=0$. Accordingly, $\mathcal{K}(p, k)$ is in $\mathbb{Z}_{p}$ for all $k<(p+3) / 2$ by definition.

Proof of Lemma \%. For all $1 \leq j \leq n-1$ we note that $\nu_{p}((2 n+3) ! !) \geq \nu_{p}((2 j+5) ! !)$ and thus $\mathcal{F}_{n}^{\prime} \in \mathbb{Z}_{p}$. Put

$$
q_{k}(j):=\left\{\begin{array}{l}
1, \text { if } k=0 \\
\prod_{i=1}^{k}(2(j+i)), \text { if } k \geq 1
\end{array}\right.
$$

Then by definition

$$
\mathcal{K}(p, k)=\sum_{j=1}^{p-1} \frac{(2 j) ! !}{(2 j+5) ! !} q_{k}(j) .
$$

From the definition of $q_{k}(j)$ we have $\operatorname{deg}\left(q_{k}(j)\right)=k$, and we write $q_{k}(j)=b_{k}^{(k)} j^{k}+b_{k-1}^{(k)} j^{k-1}+$ $\cdots+b_{1}^{(k)} j+b_{0}^{(k)}$. Moreover, we define

$$
\mathcal{G}_{k}:=\sum_{j=1}^{p-1} j^{k} \frac{(2 j) ! !}{(2 j+5) ! !} .
$$

Hence,

$$
\begin{aligned}
\mathcal{K}(p, k) & =\sum_{j=1}^{p-1} \frac{(2 j) ! !}{(2 j+5) ! !}\left(b_{k}^{(k)} j^{k}+\cdots+b_{0}^{(k)}\right) \\
& =b_{k}^{(k)} \mathcal{G}_{k}+\cdots+b_{0}^{(k)} \mathcal{G}_{0} .
\end{aligned}
$$

Assume that $k<(p+3) / 2$. Then by Lemma 9 we have

$$
0 \equiv p \mathcal{K}(p, k) \equiv p\left(b_{k}^{(k)} \mathcal{G}_{k}+\cdots+b_{0}^{(k)} \mathcal{G}_{0}\right) \quad \bmod p \mathbb{Z}_{p}
$$

We know by Lemma 9 that $\widetilde{p \mathcal{G}}_{0}=0$. Consequently, for $k=1$ (3.7) implies that $\widetilde{p \mathcal{G}}_{1}=0$ independently of $b_{1}^{(1)}$. Inductively $\widetilde{p \mathcal{G}}_{k}=0$ for all $k<(p+3) / 2$ independently of the coefficient $b_{k}^{(k)}$. Accordingly, (3.7) holds even if the polynomial $q_{k}(j)$ is interchanged by any polynomial $q$ of degree strictly less than $(p+3) / 2$. This completes the proof of Lemma 7 .

Proof of Lemma 8. By interchanging the order in the summations in $\mathcal{F}_{n}^{\prime \prime}$ we obtain

$$
\mathcal{F}_{n}^{\prime \prime}=(2 n+3) ! ! \sum_{i=1}^{n-1} f(i) \sum_{j=i+1}^{n-1} \frac{(2 j+4) ! !}{(2 j+5) ! !} .
$$


From Lemma 4 we have

$$
\begin{aligned}
\sum_{j=i+1}^{n-1} \frac{(2 j+4) ! !}{(2 j+5) ! !} & =\sum_{j=1}^{n-1} \frac{(2 j+4) ! !}{(2 j+5) ! !}-\sum_{j=1}^{i} \frac{(2 j+4) ! !}{(2 j+5) ! !} \\
& =\frac{(2 n+4) ! !-2(2 n+3) ! !}{(2 n+3) ! !}-\frac{(2 i+6) ! !-2(2 i+5) ! !}{(2 i+5) ! !} .
\end{aligned}
$$

Insertion of (3.9) in (3.8) yields

$$
\begin{aligned}
\mathcal{F}_{n}^{\prime \prime}= & (2 n+3) ! ! \sum_{i=1}^{n-1} f(i)\left(\frac{(2 n+4) ! !-2(2 n+3) ! !}{(2 n+3) ! !}-\frac{(2 i+6) ! !-2(2 i+5) ! !}{(2 i+5) ! !}\right) \\
= & ((2 n+4) ! !-2(2 n+3) ! !) \sum_{i=1}^{n-1} f(i) \\
& -(2 n+3) ! ! \sum_{i=1}^{n-1} f(i) \frac{(2 i+6) ! !-2(2 i+5) ! !}{(2 i+5) ! !} \\
= & (2 n+4) ! ! \sum_{i=1}^{n-1} f(i)-(2 n+3) ! ! \sum_{i=1}^{n-1} f(i) \frac{(2 i+6) ! !}{(2 i+5) ! !} .
\end{aligned}
$$

The upcoming Lemma 10, Lemma 12 and Lemma 13 address the reductions of the specific functions that are considered in the proof of the Main Lemma.

Lemma 10. Let $p$ be an odd prime. For each integer $n \geq 1$, let $\mathcal{U}_{n}, \mathcal{V}_{n}, \mathcal{W}_{n}$ and $\mathcal{X}_{n}$ in $\mathbb{Q}_{p}$ be defined by

$$
\begin{aligned}
\mathcal{U}_{n}(a, b) & :=(2 n+2) ! ! \sum_{j=1}^{n-1} \frac{\mathcal{S}_{j}(a, b)(2 j+3)}{(2 j+4) ! !}, \\
\mathcal{V}_{n}(a, b, c) & :=(2 n+2) ! ! \sum_{j=1}^{n-1} \frac{\mathcal{R}_{j} \cdot\left(a j^{2}+b j+c\right)}{(2 j+4) ! !}, \\
\mathcal{W}_{n} & :=(2 n+2) ! ! \sum_{j=1}^{n-1} \frac{\mathcal{T}_{j} \cdot(2 j+3)}{(2 j+4) ! !},
\end{aligned}
$$

and

$$
\mathcal{X}_{n}(a, b):=(2 n+2) ! ! \sum_{j=1}^{n-1} \frac{(a j+b)(2 j+1) ! !}{(2 j+4) ! !}
$$

Then

$$
\widetilde{\mathcal{U}}_{p}(a, b)=3 / 2 a-3 b, \quad \widetilde{\mathcal{V}}_{p}(a, b, c)=-3 a+b, \quad \widetilde{\mathcal{W}}_{p}=2 \text { and } \widetilde{\mathcal{X}}_{p}=0
$$

Proof. The proof relies on repeated use of Lemma 6. For $\mathcal{U}_{n}$ we have

$$
\widetilde{\mathcal{U}}_{p}(a, b)=2(2 p-1) \widetilde{\mathcal{S}}_{p-2}(a, b)+(2 p+1) \widetilde{\mathcal{S}}_{p-1}(a, b)=\widetilde{\mathcal{S}}_{p-1}(a, b)-2 \widetilde{\mathcal{S}}_{p-2}(a, b) .
$$

By Lemma $5, \widetilde{\mathcal{S}}_{p-1}(a, b)=a / 2-b$ and $\widetilde{\mathcal{S}}_{p-2}=-a / 2+b$, so that $\widetilde{\mathcal{U}}_{p}(a, b)=3 / 2 a-3 b$.

For $\mathcal{V}_{n}$ we have

$$
\widetilde{\mathcal{V}}_{p}(a, b, c)=2(2 p-1)(4 a-2 b+c) \widetilde{\mathcal{R}}_{p-2}+(2 p+1)(a-b+c) \widetilde{\mathcal{R}}_{p-1}
$$


By Lemma 4 we obtain the reduction

$$
\widetilde{\mathcal{V}}_{p}(a, b, c)=(-2)(4 a-2 b+c)(2 p-4) ! !+(a-b+c)(2 p-2) ! ! .
$$

From Lemma 3 the reductions of $(2 p-2) ! !$ and $(2 p-4) ! !$ are -1 and $1 / 2$. Hence,

$$
\widetilde{\mathcal{V}}_{p}(a, b, c)=-3 a+b \text {. }
$$

In a similar way we deduce by Lemma 6 that for $\mathcal{W}_{n}$ we have

$$
\widetilde{\mathcal{W}}_{p}=2(2 p-1) \widetilde{\mathcal{T}}_{p-2}+(2 p+1) \widetilde{\mathcal{T}}_{p-1},
$$

and by Lemma 4 it follows that

$$
\widetilde{\mathcal{W}}_{p}=(-2)(-1)=2
$$

Finally, concerning $\mathcal{X}_{p}$ we note that

$$
\nu_{p}\left(\frac{(2 j+1) ! !}{(2 j+4) ! !}\right) \geq 0 .
$$

Hence, $\widetilde{\mathcal{X}}_{p}(a, b)=0$. This completes the proof of Lemma 10 .

Lemma 11. Let $p \geq 5$ be a prime, and $\gamma=\frac{p-3}{2}$. Furthermore let $n \geq 1$ be an integer, $\mathcal{H}_{n}$ and $\mathcal{H}_{n}^{\prime}$ in $\mathbb{Q}_{p}$ be defined as

$$
\mathcal{H}_{n}:=\sum_{j=1}^{n} \frac{1}{2 j+1}
$$

and

$$
\mathcal{H}_{n}^{\prime}:=\sum_{j=1}^{n} \frac{1}{2 j},
$$

then $\mathcal{H}_{\gamma}, \mathcal{H}_{\gamma}^{\prime} \in \mathbb{Z}_{p}$ and $\widetilde{\mathcal{H}}_{\gamma}+\widetilde{\mathcal{H}}_{\gamma}^{\prime}=0$.

Proof. By definition $\mathcal{H}_{\gamma}$ and $\mathcal{H}_{\gamma}^{\prime}$ are in $\mathbb{Z}_{p}$, and also note that the sum of the multiplicative inverse elements of 1 and $p-1$ is 0 . Thus

$$
\mathcal{H}_{\gamma}+\mathcal{H}_{\gamma}^{\prime}=\sum_{j=1}^{p-1} j^{-1} .
$$

$\mathcal{H}_{\gamma}+\mathcal{H}_{\gamma}^{\prime}$ is equal to the sum of all elements in $\mathbb{F}_{p}^{\times}$. Consequently,

$$
\mathcal{H}_{\gamma}+\mathcal{H}_{\gamma}^{\prime} \equiv 0 \quad(\bmod p)
$$

Lemma 12. Let $p \geq 7$ be a prime. For each $n \geq 1$ define $\widehat{\mathcal{S}}_{n}, \widehat{\mathcal{T}}_{n}, \widehat{\mathcal{R}}_{n}$, and $\mathcal{Z}_{n}$ in $\mathbb{Z}_{p}$ as

$$
\begin{gathered}
\widehat{\mathcal{S}}_{n}(a, b, c):=(2 n+3) ! ! \sum_{j=1}^{n-1} \frac{(c j+1)(2 j+3)}{(2 j+5) ! !} \mathcal{S}_{j}(a, b), \\
\widehat{\mathcal{T}}_{n}(a):=(2 n+3) ! ! \sum_{j=1}^{n-1} \frac{(a j+1)(2 j+3)}{(2 j+5) ! !} \mathcal{T}_{j}, \\
\widehat{\mathcal{R}}_{n}(a):=(2 n+3) ! ! \sum_{j=1}^{n-1} \frac{(2 j+2)(a j+1)}{(2 j+5) ! !} \mathcal{R}_{j},
\end{gathered}
$$




$$
\mathcal{Z}_{n}(a, b, c):=(2 n+3) ! ! \sum_{j=1}^{n-1} \frac{a j^{2}+b j+c}{(2 j+3)(2 j+5)} .
$$

Then

$$
\begin{aligned}
& \widetilde{\widehat{\mathcal{S}}}_{p}(a, b, c)=1 / 4(a(17-41 c)+b(-4+7 c)), \quad \widetilde{\widehat{\mathcal{T}}}_{p}(a)=6-15 a \\
& \widetilde{\widehat{\mathcal{R}}}(a)_{p}=9 a-3, \text { and } \widetilde{\mathcal{Z}}(a, b, c)_{p}=6 a-\frac{3}{2} b .
\end{aligned}
$$

Proof. Proof of $\widehat{\mathcal{S}}_{n}$ : By the definition of $\mathcal{S}_{j}$ we obtain

$$
\widehat{\mathcal{S}}_{n}(a, b, c)=(2 n+3) ! ! \sum_{j=1}^{n-1} \frac{c j+1}{2 j+5} \sum_{i=1}^{j} \frac{a i+b}{2 i+1} .
$$

Let $p \geq 7$ and put $n=p$. Put

$$
\mathcal{Y}_{1}:=(2 p+3) ! !\left(\frac{c(p-5)+2}{2 p} \sum_{i=1}^{(p-5) / 2} \frac{a i+b}{2 i+1}\right),
$$

and

$$
\mathcal{Y}_{2}:=(2 p+3) ! !\left(\frac{(a(p-1)+2 b}{2 p} \sum_{j=(p-1) / 2}^{p-1} \frac{c j+1}{2 j+5}\right) .
$$

Note that the only poles of $\widehat{\mathcal{S}}_{p}(a, b, c)$ occur for $j=(p-5) / 2$ and $i=(p-1) / 2$. Accordingly by Lemma 2

$$
\widehat{\mathcal{S}}_{p}(a, b, c) \equiv \mathcal{Y}_{1}+\mathcal{Y}_{2} \quad \bmod p \mathbb{Z}_{p}
$$

To finish the proof is to show that $\widetilde{\mathcal{Y}}_{1}+\widetilde{\mathcal{Y}}_{2}=1 / 4(a(17-41 c)+b(-4+7 c))$.

For the sum in (3.18) we note that

$$
\sum_{i=1}^{(p-5) / 2} \frac{a i+b}{2 i+1}=\sum_{i=1}^{(p-3) / 2} \frac{a i+b}{2 i+1}-\frac{a(p-3)+2 b}{2 p-4} .
$$

Moreover,

$$
\begin{aligned}
\sum_{i=1}^{(p-3) / 2} \frac{a i+b}{2 i+1} & =\sum_{i=1}^{(p-3) / 2} \frac{a}{2}-\left(\frac{a}{2}-b\right) \frac{1}{2 i+1} \\
& =\frac{a(p-3)}{4}-\left(\frac{a}{2}-b\right) \sum_{i=1}^{(p-3) / 2} \frac{1}{2 i+1}
\end{aligned}
$$

By the definition of $\mathcal{H}_{\gamma}$ in Lemma 11 we then have

$$
\sum_{i=1}^{(p-3) / 2} \frac{a i+b}{2 i+1}=\frac{a(p-3)}{4}-\left(\frac{a}{2}-b\right) \mathcal{H}_{\gamma} .
$$

It follows that

$$
\mathcal{Y}_{1}=\frac{(2 p+3) ! !}{p} \cdot \frac{c(p-5)+2}{2}\left(\frac{a(p-3)}{4}-\left(\frac{a}{2}-b\right) \mathcal{H}_{\gamma}-\frac{a(p-3)+2 b}{2 p-4}\right) .
$$


By a switch of index in the sum of (3.19) we obtain

$$
\begin{aligned}
\sum_{j=(p-1) / 2}^{p-1} \frac{c j+1}{2 j+5}= & \sum_{k=1}^{(p+1) / 2} \frac{c(2 k+p-3)+2}{2(2 k+p+2)} \\
= & \left(\sum_{k=1}^{(p+3) / 2} \frac{c(2 k+p-5)+2}{2(2 k+p)}\right)-\frac{c(p-3)+2}{2 p+4} \\
= & \frac{1}{2}\left(\sum_{k=1}^{(p-3) / 2} \frac{c(2 k+p-5)+2}{2 k+p}\right) \\
& -\frac{c(p-3)+2}{2 p+4}+\frac{c(2 p-6)+2}{4 p-2}+\frac{c(2 p-4)+2}{4 p+2}+\frac{c(2 p-2)+2}{4 p+6} .
\end{aligned}
$$

Put

$$
\mathcal{P}:=\frac{1}{2} \sum_{k=1}^{(p-3) / 2} \frac{c(2 k+p-5)+2}{2 k+p}=\frac{1}{2} \sum_{k=1}^{(p-3) / 2}\left(c+\frac{2-5 c}{2 k+p}\right)=\frac{c(p-3)}{4}+\frac{2-5 c}{2} \sum_{k=1}^{(p-3) / 2} \frac{1}{2 k+p} .
$$

and

$$
\sigma:=-\frac{c(p-3)+2}{2 p+4}+\frac{c(2 p-6)+2}{4 p-2}+\frac{c(2 p-4)+2}{4 p+2}+\frac{c(2 p-2)+2}{4 p+6} .
$$

Note that by definition

$$
\mathcal{Y}_{2}=+\frac{(2 p+3) ! !}{p} \cdot \frac{a(p-1)+2 b}{2}(\mathcal{P}+\sigma) .
$$

In view of Lemma 11 we have

$$
\widetilde{\mathcal{P}}=-\frac{1}{2}\left(3 / 2 c+(5 c-2) \widetilde{\mathcal{H}}_{\gamma}^{\prime}\right) .
$$

We also note that $\widetilde{\sigma}=(17 c-2) / 12$.

Together with $(3.22),(3.23)$ and using that the reduction of $(2 p+3) ! ! / p$ is -3 , we obtain

$$
\begin{aligned}
\widetilde{\mathcal{Y}}_{1}+\widetilde{\mathcal{Y}}_{2}= & -\frac{3}{2}(2-5 c)\left(-\frac{3 a}{4}-\left(\frac{a}{2}-b\right) \widetilde{\mathcal{H}}_{\gamma}-\frac{3 a}{4}+\frac{b}{2}\right) \\
& +(-3)\left(-\frac{a}{2}+b\right)\left(-\frac{3}{4} c-\frac{2 c-5}{2} \widetilde{\mathcal{H}}_{\gamma}^{\prime}+\frac{17 c-2}{12}\right) \\
= & -\frac{3}{2}(2-5 c)\left(-\frac{3}{2} a+\frac{b}{2}\right)+3\left(\frac{a}{2}-b\right)\left(\frac{2}{3} c-\frac{1}{6}\right) \\
& +\frac{3}{2}(2-5 c)\left(\frac{a}{2}-b\right)\left(\widetilde{\mathcal{H}}_{\gamma}+\widetilde{\mathcal{H}}_{\gamma}^{\prime}\right) .
\end{aligned}
$$

Finally using Lemma 11 we have

$$
\widetilde{\mathcal{Y}}_{1}+\widetilde{\mathcal{Y}}_{2}=\frac{1}{4}(a(17-41 c)+b(-4+7 c)) .
$$

In view of $(3.20)$ we have $\widetilde{\widehat{\mathcal{S}}}_{p}(a, b, c)=1 / 4(a(17-41 c)+b(-4+7 c))$ as required. 
Proof of $\widehat{\mathcal{T}}_{n}:$ From Lemma 4 we have $\mathcal{T}_{n}=(2 n+2) ! !-2(2 n+1)$ !! and we obtain

$$
\begin{aligned}
\widehat{\mathcal{T}}_{n}(a)= & (2 n+3) ! ! \sum_{j=1}^{n-1} \frac{a j+1}{(2 j+5) ! !}(2 j+3)((2 j+2) ! !-2(2 j+1) ! !) \\
= & (2 n+3) ! ! \sum_{j=1}^{n-1} \frac{(2 j) ! !}{(2 j+5) ! !}(a j+1)(2 j+3)(2 j+2) \\
& -2(2 n+3) ! ! \sum_{j=1}^{n-1} \frac{a j+1}{2 j+5} .
\end{aligned}
$$

Let $p \geq 7$ be a prime and put $n=p$. Then the reduction of the first sum is zero by Lemma 7 . The second sum has a simple pole at $j=(p-5) / 2$. Hence, by Lemma 2

$$
\widehat{\mathcal{T}}_{p}(a) \equiv-\frac{2(2 p+3) ! !}{p} \cdot \frac{a(p-5)+2}{2} \bmod p \mathbb{Z}_{p} .
$$

The corresponding reduction is then

$$
\widetilde{\widehat{\mathcal{T}}}_{p}(a)=(-2)(-3)\left(\frac{2-5 a}{2}\right)=6-15 a .
$$

Proof of $\widehat{\mathcal{R}}_{n}$ : From Lemma 4 we have $\mathcal{R}_{n}=(2 n+1) ! !-(2 n) !$ ! and we obtain

$$
\begin{aligned}
\widehat{\mathcal{R}}_{n}(a)= & (2 n+3) ! ! \sum_{j=1}^{n-1} \frac{2 j+2}{(2 j+5) ! !}(a j+1)((2 j+1) ! !-(2 j) ! !) \\
= & (2 n+3) ! ! \sum_{j=1}^{n-1} \frac{(2 j+2)(a j+1)}{(2 j+3)(2 j+5)} \\
& -(2 n+3) ! ! \sum_{j=1}^{n-1} \frac{(2 j) ! !}{(2 j+5) ! !}(a j+1)(2 j+2) .
\end{aligned}
$$

Let $p \geq 7$, and $n=p$. Then the reduction of the second sum is zero by Lemma 7 . The only poles of the first sum occur at $j=(p-5) / 2$ and $j=(p-3) / 2$. Hence, by Lemma 2

$$
\widehat{\mathcal{R}}_{p}(a) \equiv \frac{(2 p+3) ! !}{p}\left(\frac{(p-3)(a(p-5)+2)}{2(p-2)}+\frac{(p-1)(a(p-3)+2)}{2(p+2)}\right) \quad \bmod p \mathbb{Z}_{p} .
$$

The corresponding reduction is then

$$
\widetilde{\widehat{\mathcal{R}}}_{p}(a)=(-3)\left(\frac{-12 a+4}{4}\right)=9 a-3 .
$$

Proof of $\mathcal{Z}_{n}$ : Let $p \geq 7$ and $n=p$. Then we note that $\mathcal{Z}_{p}$ has two simple poles occurring at $j=(p-3) / 2$ and $j=(p-5) / 2$. Hence, by Lemma 2 we have

$$
\mathcal{Z}_{p}(a, b, c) \equiv \frac{(2 p+3) ! !}{p}\left(\frac{a(p-3)^{2}+2 b(p-3)+4 c}{4(p+2)}+\frac{a(p-5)^{2}+2 b(p-5)+4 c}{4(p-2)}\right) \bmod p \mathbb{Z}_{p} .
$$

The corresponding reduction is then

$$
\widetilde{\mathcal{Z}}_{p}(a, b, c)=(-3)\left(\frac{-16 a+4 b}{8}\right)=6 a-\frac{3}{2} b .
$$

This completes the proof of Lemma 12 . 
Lemma 13. Let $p \geq 7$ be a prime. For each integer $n \geq 1$ we define $\widehat{\mathcal{U}}_{n}, \widehat{\mathcal{V}}_{n}, \widehat{\mathcal{W}}_{n}$ and $\widehat{\mathcal{X}}_{n}$ in $\mathbb{Q}_{p}$ as

$$
\begin{gathered}
\widehat{\mathcal{U}}_{n}(a, b):=(2 n+3) ! ! \sum_{j=1}^{n-1} \frac{(2 j+4)}{(2 j+5) ! !} \mathcal{U}_{j}(a, b) \\
\widehat{\mathcal{V}}_{n}(a, b, c):=(2 n+3) ! ! \sum_{j=1}^{n-1} \frac{(2 j+4)}{(2 j+5) ! !} \mathcal{V}_{j}(a, b, c) \\
\widehat{\mathcal{W}}_{n}:=(2 n+3) ! ! \sum_{j=1}^{n-1} \frac{(2 j+4)}{(2 j+5) ! !} \mathcal{W}_{j},
\end{gathered}
$$

and

$$
\widehat{\mathcal{X}}_{n}(a, b):=(2 n+3) ! ! \sum_{j=1}^{n-1} \frac{(2 j+4)}{(2 j+5) ! !} \mathcal{X}_{j}(a, b) .
$$

Then

$$
\widetilde{\widehat{\mathcal{U}}}_{p}(a, b)=a-\frac{19}{2} b, \quad \widetilde{\widehat{\mathcal{V}}}_{p}(a, b, c)=-\frac{45}{4} a+b+3 c, \quad \widetilde{\widehat{\mathcal{W}}}_{p}=2 \text { and } \widetilde{\widehat{\mathcal{X}}}_{p}(a, b)=-3(a-b) .
$$

Proof. Proof of $\widetilde{\mathcal{U}}_{p}$ : From the definition of $\mathcal{U}_{n}$ in (3.10) and $\mathcal{S}_{n}$ in (3.3) and by applying Lemma 8 we obtain

$$
\begin{aligned}
\widehat{\mathcal{U}}_{n}(a, b) & =(2 n+3) ! ! \sum_{j=1}^{n-1} \frac{(2 j+4) ! !}{(2 j+5) ! !} \sum_{i=1}^{j-1} \frac{(2 j+3) \mathcal{S}_{j}(a, b)}{(2 j+4) ! !} \\
& =(2 n+4) ! ! \sum_{i=1}^{n-1} \frac{(2 i+3) \mathcal{S}_{i}(a, b)}{(2 i+4) ! !}-(2 n+3) ! ! \sum_{i=1}^{n-1} \frac{(2 i+3) \mathcal{S}_{i}(a, b)}{(2 i+4) ! !} \cdot \frac{(2 i+6) ! !}{(2 i+5) ! !} \\
& =(2 n+4) ! ! \sum_{i=1}^{n-1} \frac{(2 i+3) ! !}{(2 i+4) ! !} \sum_{k=1}^{i} \frac{a k+b}{2 k+1}-(2 n+3) ! ! \sum_{i=1}^{n-1} \frac{(2 i+6)}{(2 i+5)} \sum_{k=1}^{i} \frac{a k+b}{2 k+1} .
\end{aligned}
$$

Let $S_{1}$ and $S_{2}$ denote the two sums in (3.31). Let $p \geq 7$ and put $n=p$. Then we note that $\nu_{p}\left(S_{1}\right) \geq 1$ for all $i$ and $k$ except for $i \in[p-2, p-1]$ and $k=(p-1) / 2$. In view of Lemma 2 this yields,

$$
S_{1} \equiv \frac{(2 p+4) ! !}{p}\left(\frac{(2 p-1) ! !}{(2 p) ! !}\left(a\left(\frac{p-1}{2}\right)+b\right)+\frac{(2 p+1) ! !}{(2 p+2) ! !}\left(a\left(\frac{p-1}{2}\right)+b\right)\right) \bmod p \mathbb{Z}_{p} .
$$

Using Lemma 3 we obtain

$$
\widetilde{S}_{1}=-16\left(\frac{1}{2}(-a / 2+b)+\frac{1}{4}(-a / 2+b)\right)=6 a-12 b .
$$

Concerning $S_{2}$, we put

$$
\mathcal{Y}_{1}:=\frac{(2 p+3) ! !}{p}(p+1) \sum_{k=1}^{(p-5) / 2} \frac{a k+b}{2 k+1}
$$

and

$$
\mathcal{Y}_{2}:=\frac{(2 p+3) ! !}{p}\left(a\left(\frac{p-1}{2}\right)+b\right) \sum_{i=(p-1) / 2}^{p-1} \frac{2 i+6}{2 i+5}
$$


Then we note that $S_{2} \equiv \mathcal{Y}_{1}+\mathcal{Y}_{2} \bmod p \mathbb{Z}_{p}$. Using (3.21) in the proof of Lemma 12 we have

$$
\sum_{k=1}^{(p-5) / 2} \frac{a k+b}{2 k+1}=\frac{a(p-3)}{4}-\left(\frac{a}{2}-b\right) \mathcal{H}_{\gamma}-\frac{a(p-3)+2 b}{2 p-4} .
$$

It follows that

$$
\mathcal{Y}_{1}=\frac{(2 p+3) ! !}{p}(p+1)\left(\frac{a(p-3)}{4}-\left(\frac{a}{2}-b\right) \mathcal{H}_{\gamma}-\frac{a(p-3)+2 b}{2 p-4}\right) .
$$

By a switch of index in the sum in $\mathcal{Y}_{2}$ we obtain

$$
\begin{aligned}
\sum_{i=(p-1) / 2}^{p-1} \frac{2 i+6}{2 i+5} & =\sum_{i=(p-1) / 2}^{p-1} 1+\frac{1}{2 i+5} \\
& =\sum_{j=1}^{(p+1) / 2} 1+\frac{1}{2 j+p+2} \\
& =\frac{p+1}{2}+\sum_{j=1}^{(p+1) / 2} \frac{1}{2 j+p+2} \\
& =\frac{p+1}{2}-\frac{1}{p+2}+\sum_{j=1}^{(p+3) / 2} \frac{1}{2 j+p} .
\end{aligned}
$$

By fixing the index in (3.33) to fit the conditions of Lemma 11 we obtain

$$
\begin{aligned}
\sum_{i=(p-1) / 2}^{p-1} \frac{2 i+6}{2 i+5} & =\frac{p+1}{2}-\frac{1}{p+2}+\sum_{j=1}^{(p+3) / 2} \frac{1}{2 j+p} \\
& =\frac{p+1}{2}-\frac{1}{p+2}+\frac{1}{2 p+3}+\frac{1}{2 p+1}+\frac{1}{2 p-1}+\sum_{j=1}^{(p-3) / 2} \frac{1}{2 j+p} .
\end{aligned}
$$

Put

$$
\sigma^{\prime}:=\frac{p+1}{2}-\frac{1}{p+2}+\frac{1}{2 p+3}+\frac{1}{2 p+1}+\frac{1}{2 p-1} .
$$

Then by definition we have

$$
\mathcal{Y}_{2}=\frac{(2 p+3) ! !}{p}\left(a\left(\frac{p-1}{2}\right)+b\right)\left(\sigma^{\prime}+\sum_{j=1}^{(p-3) / 2} \frac{1}{2 j+p}\right) .
$$

We note that $\widetilde{\sigma^{\prime}}=1 / 3$, and together with (3.32) and (3.34) we then have

$$
\begin{aligned}
\widetilde{S}_{2}= & \widetilde{\mathcal{Y}}_{1}+\widetilde{\mathcal{Y}}_{2} \\
= & (-3)\left(\frac{-3 a}{4}-\left(\frac{a}{2}-b\right) \widetilde{\mathcal{H}}_{\gamma}+\frac{-3 a+2 b}{4}\right) \\
& +(-3)\left(-\frac{a}{2}+b\right)\left(\widetilde{\sigma}^{\prime}+\widetilde{\mathcal{H}}_{\gamma}^{\prime}\right) \\
= & 5 a-\frac{5}{2} b+\left(\frac{3}{2} a-3 b\right)\left(\widetilde{\mathcal{H}}_{\gamma}+\widetilde{\mathcal{H}}_{\gamma}^{\prime}\right) \\
= & 5 a-\frac{5}{2} b .
\end{aligned}
$$


Thus, from (3.31) we obtain

$$
\widetilde{\widehat{\mathcal{U}}}_{p}(a, b)=\widetilde{S}_{1}-\widetilde{S}_{2}=6 a-12 b-5 a+\frac{5}{2} b=a-\frac{19}{2} b .
$$

Proof of $\widetilde{\widehat{V}}_{p}$ : We recall the definition of $\mathcal{V}_{j}(a, b, c)$ in $(3.11)$ and then apply Lemma 8 to obtain

$$
\begin{aligned}
\widehat{\mathcal{V}}_{n}(a, b, c) & =(2 n+3) ! ! \sum_{j=1}^{n-1} \frac{(2 j+4) ! !}{(2 j+5) ! !} \sum_{i=1}^{j-1} \frac{\mathcal{R}_{j}\left(a i^{2}+b i+c\right)}{(2 j+4) ! !} \\
& =(2 n+4) ! ! \sum_{i=1}^{n-1} \frac{\mathcal{R}_{i}\left(a i^{2}+b i+c\right)}{(2 i+4) ! !}-(2 n+3) ! ! \sum_{i=1}^{n-1} \frac{\mathcal{R}_{i}\left(a i^{2}+b i+c\right)}{(2 i+4) ! !} \cdot \frac{(2 i+6) ! !}{(2 i+5) ! !} .
\end{aligned}
$$

We recall that $\mathcal{R}_{n}=(2 n+1) ! !-(2 n) !$ !. Insertion into $(3.36)$ yields

$$
\begin{aligned}
\widehat{\mathcal{V}}_{n}(a, b, c)= & (2 n+4) ! ! \sum_{i=1}^{n-1} \frac{\left((2 i+1) ! !-(2 i) ! !\left(a i^{2}+b i+c\right)\right.}{(2 i+4) ! !} \\
& -(2 n+3) ! ! \sum_{i=1}^{n-1} \frac{((2 i+1) ! !-(2 i) ! !)\left(a i^{2}+b i+c\right)}{(2 i+4) ! !} \cdot \frac{(2 i+6) ! !}{(2 i+5) ! !} \\
= & (2 n+4) ! ! \sum_{i=1}^{n-1} \frac{(2 i+1) ! !\left(a i^{2}+b i+c\right)}{(2 i+4) ! !}-(2 n+4) ! ! \sum_{i=1}^{n-1} \frac{a i^{2}+b i+c}{(2 i+2)(2 i+4)} \\
& -(2 n+3) ! ! \sum_{i=1}^{n-1} \frac{\left(a i^{2}+b i+c\right)(2 i+6)}{(2 i+3)(2 i+5)} \\
& +(2 n+3) ! ! \sum_{i=1}^{n-1} \frac{(2 i) ! !\left(a i^{2}+b i+c\right)(2 i+6)}{(2 i+5) ! !}
\end{aligned}
$$

We denote each individual sum in the above equation as $S_{1}, S_{2}, S_{3}$ and $S_{4}$ respectively. Let $p \geq 7$ and put $n=p$. Then we note that $\nu_{p}\left(\frac{(2 i+1) ! !}{(2 i+4) ! !}\right) \geq 0$, and thus the reduction of $S_{1}$ is 0 . However $S_{2}$ and $S_{3}$ has simple poles occurring at $i=p-1$ and $i=p-2$ and $i=(p-3) / 2$ and $i=(p-5) / 2$ respectively. By Lemma 2 this yields

$$
S_{2} \equiv \frac{(2 p+4) ! !}{4 p}\left(\frac{a(p-2)^{2}+b(p-2)+c}{p-1}+\frac{a(p-1)^{2}+b(p-1)+c}{p+1}\right) \quad \bmod p \mathbb{Z}_{p},
$$

and

$$
S_{3} \equiv \frac{(2 p+3) ! !}{p}\left(\frac{\left(a\left(\frac{p-3}{2}\right)^{2}+b\left(\frac{p-3}{2}\right)+c\right)(p+3)}{p+2}+\frac{\left(a\left(\frac{p-5}{2}\right)^{2}+b\left(\frac{p-5}{2}\right)+c\right)(p+1)}{p-2}\right) \bmod p \mathbb{Z}_{p} .
$$

The corresponding reductions are

$$
\widetilde{S}_{2}=-4(-3 a+b)=12 a-4 b
$$

and

$$
\widetilde{S}_{3}=-3\left(\frac{1}{4} a-b+c\right)=-\frac{3}{4} a+3 b-3 c .
$$

Finally we have $\widetilde{S}_{4}=0$ by Lemma 7 . Hence,

$$
\widetilde{\widehat{\mathcal{V}}}_{p}(a, b, c)=-\left(\widetilde{S}_{2}+\widetilde{S}_{3}\right)=-\frac{45}{4} a+b+3 c .
$$


Proof of $\widetilde{\mathcal{W}}_{p}$ : From the definition of $\mathcal{W}_{n}$ in $(3.12)$ and $\mathcal{T}_{n}$ in $(3.2)$ and Lemma 8 we obtain

$$
\begin{aligned}
\widehat{\mathcal{W}}_{n}= & (2 n+3) ! ! \sum_{j=1}^{n-1} \frac{(2 j+4) ! !}{(2 j+5) ! !} \sum_{i=1}^{j-1} \frac{(2 i+3) \mathcal{T}_{i}}{(2 i+4) ! !} \\
= & (2 n+4) ! ! \sum_{i=1}^{n-1} \frac{(2 i+3) \mathcal{T}_{i}}{(2 i+4) ! !}-(2 n+3) ! ! \sum_{i=1}^{n-1} \frac{(2 i+3) \mathcal{T}}{(2 i+4) ! !} \cdot \frac{(2 i+6) ! !}{(2 i+5) ! !} \\
= & (2 n+4) ! ! \sum_{i=1}^{n-1} \frac{(2 i+3)((2 i+2) ! !-2(2 i+1) ! !)}{(2 i+4) ! !} \\
& -(2 n+3) ! ! \sum_{i=1}^{n-1} \frac{(2 i+3)((2 i+2) ! !-2(2 i+1) ! !)}{(2 i+4) ! !} \cdot \frac{(2 i+6) ! !}{(2 i+5) ! !} \\
= & (2 n+4) ! ! \sum_{i=1}^{n-1} \frac{2 i+3}{2 i+4}-2(2 n+4) ! ! \sum_{i=1}^{n-1} \frac{(2 i+3)(2 i+1) ! !}{(2 i+4) ! !} \\
& -(2 n+3) ! ! \sum_{i=1}^{n-1}(2 i+3)(2 i+6)(2 i+2) \cdot \frac{(2 i) ! !}{(2 i+5) ! !}+2(2 n+3) ! ! \sum_{i=1}^{n-1} \frac{2 i+6}{2 i+5} .
\end{aligned}
$$

Let $p \geq 7$ and put $n=p$, and then let each of the sums the last equality of (3.37) be denoted $S_{1}, S_{2}, S_{3}$ and $S_{4}$ respectively. First we note that $\widetilde{S}_{3}=0$ by Lemma 7 . Secondly, $S_{2}$ has no poles. Thus, $\widetilde{S}_{2}=0$. Hence, $\widetilde{\mathcal{W}}_{p}=\widetilde{S}_{1}+\widetilde{S}_{4}$. Note that $S_{1}$ and $S_{4}$ have simple poles, occurring at $i=p-2$ and $i=(p-5) / 2$ respectively. This yields

$$
S_{1} \equiv \frac{(2 p+4) ! !}{p} \cdot \frac{2 p-1}{2} \bmod p \mathbb{Z}_{p}
$$

and

$$
S_{4} \equiv \frac{2(2 p+3) ! !}{p}(p+1) \quad \bmod p \mathbb{Z}_{p}
$$

from which we deduce that

$$
\widetilde{\widehat{\mathcal{W}}}_{p}=\widetilde{S}_{1}+\widetilde{S}_{4}=8-6=2
$$

Proof of $\widetilde{\mathcal{X}}_{p}$ : Using the definition of $\mathcal{X}_{n}$ in (3.13) and Lemma 8 we have

$$
\begin{aligned}
\widehat{\mathcal{X}}_{n}(a, b) & =(2 n+3) ! ! \sum_{j=1}^{n-1} \frac{(2 j+4) ! !}{(2 j+5) ! !} \sum_{i=1}^{j-1} \frac{(a i+b)(2 i+1) ! !}{(2 i+4) ! !} \\
& =(2 n+4) ! ! \sum_{i=1}^{n-1} \frac{(a i+b)(2 i+1) ! !}{(2 i+4) ! !}-(2 n+3) ! ! \sum_{i=1}^{n-1} \frac{(a i+b)(2 i+1) ! !}{(2 i+4) ! !} \cdot \frac{(2 i+6) ! !}{(2 i+5) ! !} \\
& =(2 n+4) ! ! \sum_{i=1}^{n-1} \frac{(a i+b)(2 i+1) ! !}{(2 i+4) ! !}-(2 n+3) ! ! \sum_{i=1}^{n-1} \frac{(a i+b)(2 i+6)}{(2 i+3)(2 i+5)} .
\end{aligned}
$$

Let $p \geq 7$ and put $n=p$. The first sum in (3.39) contains no poles and therefore has reduction zero. The second sum has simple poles at $i=(p-3) / 2$ and $i=(p-5) / 2$. Then by Lemma 2

$$
\widehat{\mathcal{X}}_{p}(a, b) \equiv-\frac{(2 p+3) ! !}{p}\left(\frac{\left(a\left(\frac{p-3}{2}\right)+b\right)(p+3)}{p+2}+\frac{\left(a\left(\frac{p-5}{2}\right)+b\right)(p+1)}{p-2}\right) \quad \bmod p \mathbb{Z}_{p} .
$$


This corresponds to the reduction

$$
\widetilde{\widehat{\mathcal{X}}}_{p}(a, b)=-3(a-b)
$$

An important part of the proof of the Main Lemma is our ability to explicitly express the solutions of the difference equations, and thus the following lemma from [Ela05] is of importance.

Lemma 14. [Ela05, §1.2] Let $k$ be a field. Furthermore let $f, g: \mathbb{Z}^{+} \rightarrow k$, and $y_{0} \in k$. Given a nonhomogeneous difference equation

$$
y_{n+1}=f(n) y_{n}+g(n), y_{n_{0}}=y_{0}
$$

where $n_{0} \in[0, n]$. The general solution to the difference equation is given by

$$
y_{n}=\left[\prod_{j=n_{0}}^{n-1} f(j)\right] y_{0}+\sum_{r=n_{0}}^{n-1}\left[\prod_{j=r+1}^{n-1} f(j)\right] g(r) .
$$

\section{Main Lemma and proof of Theorem B}

In this section we state and prove our Main Lemma, and give the proof of Theorem B. For convenience we give the following definition. Given a ring $R$ and an element $a \in R$ we let $\langle a\rangle$ denote the ideal of $R$ generated by $a$.

Main Lemma. Let $p$ be an odd prime and let $k$ be a field of characteristic $p$. Let $f \in k[[\zeta]]$ be defined as

$$
f(\zeta)=\zeta\left(1+\sum_{j=2}^{+\infty} a_{j} \zeta^{j}\right)
$$

Let $\alpha_{1}, \beta_{1}$ and $\gamma_{1}$ be defined as follows

$$
\alpha_{1}:=a_{2}^{p-2}\left(\frac{3}{2} a_{2}^{3}+a_{3}^{2}-a_{2} a_{4}\right), \quad \beta_{1}:=\frac{a_{3}}{a_{2}} \alpha_{1}, \quad \gamma_{1}:=-\left(\frac{3 a_{2}}{2}-\frac{a_{4}}{a_{2}}\right) \alpha_{1} .
$$

Then

$$
f^{p}(\zeta) \equiv \zeta+\alpha_{1} \zeta^{2 p+3}+\beta_{1} \zeta^{2 p+4}+\gamma_{1} \zeta^{2 p+5} \bmod \left\langle\zeta^{2 p+6}\right\rangle
$$

Proof of Main Lemma. Analogous to [LRL16b] for $m=1$ we define the recurrence relation $\Delta_{1}(\zeta):=f(\zeta)-\zeta$ and for $m \geq 2$

$$
\Delta_{m}(\zeta):=\Delta_{m-1}(f(\zeta))-\Delta_{m-1}(\zeta) .
$$

Note that $\Delta_{p}(\zeta)=f^{p}(\zeta)-\zeta$, and $\operatorname{ord}\left(\Delta_{m}\right) \geq 2 m+1$.

For technical reasons we define $F_{\ell}:=\mathbb{Q}_{p}\left[x_{1}, x_{2}, \ldots, x_{\ell}\right]$, and $F_{\infty}:=\mathbb{Q}_{p}\left[x_{1}, x_{2}, \ldots\right]$. Moreover we consider the power series $\widehat{f} \in F_{\infty}[[\zeta]]$ defined as

$$
\widehat{f}(\zeta):=\zeta\left(1+\sum_{j=1}^{+\infty} x_{j} \zeta^{j+1}\right)
$$

For $m=1$ we define the relation $\widehat{\Delta}_{1}(\zeta):=\widehat{f}(\zeta)-\zeta$ and for each integer $m \geq 2$

$$
\widehat{\Delta}_{m}(\zeta):=\widehat{\Delta}_{m-1}(\widehat{f}(\zeta))-\widehat{\Delta}_{m-1}(\zeta) .
$$

Defined in this way there is a clear relation between $f(\zeta)$ and $\widehat{f}(\zeta)$ and thus between $\Delta_{m}$ and $\widehat{\Delta}_{m}$. In the last part of the proof we exploit this relation to find the coefficients of $f^{p}(\zeta)-\zeta$. 
Let $x=x_{\infty}:=\left(x_{1}, x_{2}, \ldots\right)$, and let $\mathcal{A}_{m}(x), \mathcal{B}_{m}(x), \mathcal{C}_{m}(x), \mathcal{D}_{m}(x)$ and $\mathcal{E}_{m}(x)$ be defined by

$$
\mathcal{A}_{m+1}(x)=x_{1}(2 m+1) \mathcal{A}_{m},
$$

$$
\mathcal{B}_{m+1}(x)=x_{2}(2 m+1) \mathcal{A}_{m}+x_{1}(2 m+2) \mathcal{B}_{m},
$$

$$
\mathcal{C}_{m+1}(x)=\left(x_{1}^{2} m(2 m+1)+x_{3}(2 m+1)\right) \mathcal{A}_{m}+x_{2}(2 m+2) \mathcal{B}_{m}+x_{1}(2 m+3) \mathcal{C}_{m},
$$

$$
\begin{aligned}
\mathcal{D}_{m+1}(x)= & \left(x_{4}(2 m+1)+x_{1} x_{2}(2 m+1)(2 m)\right) \mathcal{A}_{m}+\left(x_{3}(2 m+2)+x_{1}^{2}(m+1)(2 m+1)\right) \mathcal{B}_{m} \\
& +x_{2}(2 m+3) \mathcal{C}_{m}+x_{1}(2 m+4) \mathcal{D}_{m}
\end{aligned}
$$

and

$$
\begin{aligned}
\mathcal{E}_{m+1}(x)= & \left(x_{5}(2 m+1)+x_{1} x_{3}(2 m+1)(2 m)+x_{1}^{3}\left(\begin{array}{c}
2 m+1 \\
3
\end{array}\right)+m(2 m+1) x_{2}^{2}\right) \mathcal{A}_{m} \\
& +\left(x_{4}(2 m+2)+x_{1} x_{2}(2 m+2)(2 m+1)\right) \mathcal{B}_{m} \\
& +\left(x_{3}(2 m+3)+x_{1}^{2}\left(\begin{array}{c}
2 m+3 \\
2
\end{array}\right)\right) \mathcal{C}_{m}+x_{2}(2 m+4) \mathcal{D}_{m}+x_{1}(2 m+5) \mathcal{E}_{m}
\end{aligned}
$$

with initial conditions $\left(\mathcal{A}_{1}, \mathcal{B}_{1}, \mathcal{C}_{1}, \mathcal{D}_{1}, \mathcal{E}_{1}\right)=\left(x_{1}, x_{2}, x_{3}, x_{4}, x_{5}\right)$. We prove by induction that for $m \geq 1$ we have

$(4.6)$

$\widehat{\Delta}_{m}(\zeta) \equiv \mathcal{A}_{m}(x) \zeta^{2 m+1}+\mathcal{B}_{m}(x) \zeta^{2 m+2}+\mathcal{C}_{m}(x) \zeta^{2 m+3}+\mathcal{D}_{m}(x) \zeta^{2 m+4}+\mathcal{E}_{m}(x) \zeta^{2 m+5} \bmod \left\langle\zeta^{2 m+6}\right\rangle$.

Throughout the rest of the proof let $\mathcal{A}_{m}:=\mathcal{A}_{m}(x), \mathcal{B}_{m}:=\mathcal{B}_{m}(x), \mathcal{C}_{m}:=\mathcal{C}_{m}(x), \mathcal{D}_{m}:=\mathcal{D}_{m}(x)$ and $\mathcal{E}_{m}:=\mathcal{E}_{m}(x)$ unless otherwise specified. 
For $m=1$ (4.6) holds by definition. Let $m \geq 1$ be such that (4.6) holds. Then

$$
\begin{aligned}
\widehat{\Delta}_{m+1}(\zeta) \equiv & \widehat{\Delta}_{m}(\widehat{f}(\zeta))-\widehat{\Delta}_{m}(\zeta) \\
\equiv & \mathcal{A}_{m}\left[\left(\zeta+\widehat{\Delta}_{1}(\zeta)\right)^{2 m+1}-\zeta^{2 m+1}\right]+\mathcal{B}_{m}\left[\left(\zeta+\widehat{\Delta}_{1}(\zeta)\right)^{2 m+2}-\zeta^{2 m+2}\right] \\
& +\mathcal{C}_{m}\left[\left(\zeta+\widehat{\Delta}_{1}(\zeta)\right)^{2 m+3}-\zeta^{2 m+3}\right]+\mathcal{D}_{m}\left[\left(\zeta+\widehat{\Delta}_{1}(\zeta)\right)^{2 m+4}-\zeta^{2 m+4}\right] \\
& +\mathcal{E}_{m}\left[\left(\zeta+\widehat{\Delta}_{1}(\zeta)\right)^{2 m+5}-\zeta^{2 m+5}\right] \\
\equiv & \mathcal{A}_{m}\left[x_{1}(2 m+1) \zeta^{2 m+3}+x_{2}(2 m+1) \zeta^{2 m+4}+\left(x_{3}(2 m+1)+x_{1}^{2}\left(\begin{array}{c}
2 m+1 \\
2
\end{array}\right)\right) \zeta^{2 m+5}\right. \\
& +\left(x_{4}(2 m+1)+x_{1} x_{2}(2 m+1)(2 m)\right) \zeta^{2 m+6} \\
& \left.+\left(x_{5}(2 m+1)+x_{1} x_{3}(2 m+1)(2 m)+x_{1}^{3}\left(\begin{array}{c}
2 m+1 \\
3
\end{array}\right)+x_{2}^{2}\left(\begin{array}{c}
2 m+1 \\
2
\end{array}\right)\right) \zeta^{2 m+7}\right] \\
& +\mathcal{B}_{m}\left[x_{1}(2 m+2) \zeta^{2 m+4}+x_{2}(2 m+2) \zeta^{2 m+5}+\left(x_{1}^{2}\left(\begin{array}{c}
2 m+2 \\
2
\end{array}\right)+x_{3}(2 m+2)\right) \zeta^{2 m+6}\right. \\
& \left.+\left(x_{4}(2 m+2)+x_{1} x_{2}(2 m+2)(2 m+1)\right) \zeta^{2 m+7}\right] \\
& +\mathcal{C}_{m}\left[x_{1}(2 m+3) \zeta^{2 m+5}+x_{2}(2 m+3) \zeta^{2 m+6}+\left(x_{3}(2 m+3)+x_{1}^{2}\left(\begin{array}{c}
2 m+3 \\
2
\end{array}\right)\right) \zeta^{2 m+7}\right] \\
& +\mathcal{D}_{m}\left[x_{1}(2 m+4) \zeta^{2 m+6}+x_{2}(2 m+4) \zeta^{2 m+7}\right]+\mathcal{E}_{m}\left[x_{1}(2 m+5) \zeta^{2 m+7}\right] \\
\equiv & \mathcal{A}_{m+1} \zeta^{2 m+3}+\mathcal{B}_{m+1} \zeta^{2 m+4}+\mathcal{C}_{m+1} \zeta^{2 m+5}+\mathcal{D}_{m+1} \zeta^{2 m+6}+\mathcal{E}_{m+1} \zeta^{2 m+7} \bmod \left\langle\zeta^{2 m+8}\right\rangle .
\end{aligned}
$$

This completes the proof of the induction step and proves (4.6), and we note that the difference equations only depend on the coefficients $x_{1}, x_{2}, x_{3}, x_{4}, x_{5}$ and not those for higher order terms, so $\mathcal{A}_{m}, \mathcal{B}_{m}, \mathcal{C}_{m}, \mathcal{D}_{m}, \mathcal{E}_{m} \in F_{5}$.

We divide the proof of the Lemma in three cases. First we consider the cases $p=3$ and $p=5$. In the third case we prove the Lemma for all $p \geq 7$.

For the cases $p=3$ and $p=5$ we will explicitly compute the values of the coefficients in $\widehat{\Delta}_{m}$ for $m \in[1,3]$ and $m \in[1,5]$ respectively. For convenience we redefine $F_{5}$ as $\mathbb{F}_{p}\left[x_{1}, \ldots, x_{5}\right]$ for $p=3$ and $p=5$ respectively. ${ }^{\mathrm{i}}$ Thereby we also utilize the fact that we are working over a field of characteristic 3 and 5 respectively. This fact will be used continuously without comment throughout the proof.

Case 1, $p=3$. From (4.1), (4.2), (4.3), (4.4) and (4.5) we have $\mathcal{A}_{2}=0, \mathcal{A}_{3}=0$, and $\mathcal{B}_{2}=$ $x_{1} x_{2}, \mathcal{B}_{3}=0$. Furthermore, $\mathcal{C}_{2}=x_{2}^{2}+2 x_{1} x_{3}, \mathcal{C}_{3}=x_{1}\left(x_{2}^{2}+2 x_{1} x_{3}\right)=x_{1}\left(x_{2}^{2}-x_{1} x_{3}\right)$. We also have $\mathcal{D}_{2}=x_{2} x_{3}+2 x_{2} x_{3}=0, \mathcal{D}_{3}=x_{2}\left(x_{2}^{2}-x_{1} x_{3}\right)$. Finally we obtain $\mathcal{E}_{2}=x_{1} x_{5}+x_{3}\left(2 x_{3}+x_{1}^{2}\right)+x_{2} x_{4}$, and $\mathcal{E}_{3}=x_{3}\left(x_{2}^{2}-x_{1} x_{3}\right)$. Thus we have

$$
\widehat{\Delta}_{3}(\zeta) \equiv x_{1}\left(x_{2}^{2}-x_{1} x_{3}\right) \zeta^{9}+x_{2}\left(x_{2}^{2}-x_{1} x_{3}\right) \zeta^{10}+x_{3}\left(x_{2}^{2}-x_{1} x_{3}\right) \zeta^{11} \bmod \left\langle\zeta^{12}\right\rangle .
$$

For each $i \in\{1,2,3\}$ we specialize each $x_{i}$ to $a_{i+1}$ and obtain

$$
\begin{aligned}
\widehat{\Delta}_{3}(\zeta) \equiv f^{3}(\zeta)-\zeta & \equiv a_{2}\left(a_{3}^{2}-a_{2} a_{4}\right) \zeta^{9}+a_{3}\left(a_{3}^{2}-a_{2} a_{4}\right) \zeta^{10}+a_{4}\left(a_{3}^{2}-a_{2} a_{4}\right) \zeta^{11} \\
& \equiv \alpha_{1} \zeta^{9}+\beta_{1} \zeta^{10}+\gamma_{1} \zeta^{11} \bmod \left\langle\zeta^{12}\right\rangle
\end{aligned}
$$

This completes the proof of the Lemma for the case $p=3$.

\footnotetext{
iNote that $3 \mid(2 m+1)(2 m)(2 m-1)$ so $\left(\begin{array}{c}2 m+1 \\ 3\end{array}\right)$ defines an element in $\mathbb{F}_{3}$.
} 
Case 2, $p=5$. We continue in a similar procedure as for $p=3$ using (4.1), (4.2), (4.3), (4.4) and (4.5) to compute $\widehat{\Delta}_{5}$. We have

$$
\mathcal{A}_{2}=3 x_{1}^{2}, \quad \mathcal{A}_{3}=0, \quad \mathcal{A}_{4}=0, \quad \mathcal{A}_{5}=0,
$$

and

$$
\mathcal{B}_{2}=2 x_{1} x_{2}, \quad \mathcal{B}_{3}=2 x_{1}^{2} x_{2}, \quad \mathcal{B}_{4}=x_{1}^{3} x_{2}, \quad \mathcal{B}_{5}=0 .
$$

Concerning $\mathcal{C}_{m}$ we have

$$
\mathcal{C}_{2}=\left(3 x_{1}^{2}+3 x_{3}\right) \mathcal{A}_{1}+4 x_{2} \mathcal{B}_{1}=3 x_{1}^{3}+4 x_{2}^{2}+3 x_{1} x_{3}, \quad \mathcal{C}_{3}=x_{2} \mathcal{B}_{2}+2 x_{1} \mathcal{C}_{2}=x_{1}^{4}+x_{1}^{2} x_{3} .
$$

Using $\mathcal{A}_{3}=\mathcal{A}_{4}=0$ we obtain

$\mathcal{C}_{4}=8 x_{2} \mathcal{B}_{3}+9 x_{1} \mathcal{C}_{3}=x_{1}^{2} x_{2}^{2}+4 x_{1}\left(x_{1}^{4}+x_{1}^{2} x_{3}\right), \quad \mathcal{C}_{5}=10 x_{2} \mathcal{B}_{4}+11 x_{1} \mathcal{C}_{4}=x_{1}^{3}\left(4 x_{1}^{3}+x_{2}^{2}+4 x_{1} x_{3}\right)$.

Continuing for $\mathcal{D}_{m}$ we have

$$
\mathcal{D}_{2}=\left(3 x_{4}+6 x_{1} x_{2}\right) x_{1}+\left(4 x_{3}+6 x_{1}^{2}\right) x_{2}+5(\cdots) \mathcal{C}_{1}+6 x_{1} x_{4}=4 x_{1} x_{4}+2 x_{1}^{2} x_{2}+4 x_{2} x_{3} .
$$

Again using $\mathcal{A}_{3}=0$ we obtain

$$
\mathcal{D}_{4}=\left(8 x_{3}+28 x_{1}^{2}\right) \mathcal{B}_{3}+9 x_{2} \mathcal{C}_{3}+10(\cdots) \mathcal{D}_{3}=2 x_{1}^{2} x_{2}\left(8 x_{3}+28 x_{1}^{2}\right)+9 x_{2}\left(x_{1}^{4}+x_{1}^{2} x_{3}\right)=0 .
$$

Finally we have

$$
\mathcal{D}_{5}=5(\cdots) \mathcal{B}_{4}+11 x_{2} \mathcal{C}_{4}=x_{2}\left(x_{1}^{2} x_{2}^{2}+4 x_{1}\left(x_{1}^{4}+x_{1}^{2} x_{3}\right)\right)=x_{1}^{2} x_{2}\left(4 x_{1}^{3}+x_{2}^{2}+4 x_{1} x_{3}\right) .
$$

Consequently, for $\mathcal{E}_{m}$ we have

$\mathcal{E}_{2}=\left(3 x_{5}+6 x_{1} x_{3}+x_{1}^{3}+3 x_{2}\right) x_{1}+\left(4 x_{4}+12 x_{1} x_{2}\right) x_{2}+5(\cdots) x_{3}+6 x_{2} x_{4}+7 x_{1} x_{5}=x_{1}^{4}+x_{1}^{2} x_{3}$, and

$$
\begin{aligned}
\mathcal{E}_{3}= & 5(\cdots) \mathcal{A}_{2}+6 x_{2} \mathcal{B}_{2}+\left(7 x_{3}+21 x_{1}^{2}\right) \mathcal{C}_{2}+8 x_{2} \mathcal{D}_{2}+9 x_{1} \mathcal{E}_{2} \\
= & 12 x_{1} x_{2} x_{4}+\left(2 x_{3}+x_{1}^{2}\right)\left(3 x_{1}^{3}+4 x_{2}^{2}+3 x_{1} x_{3}\right) \\
& +3 x_{2}\left(4 x_{1} x_{4}+2 x_{1}^{2} x_{2}+4 x_{2} x_{3}\right)+4 x_{1}\left(x_{1}^{4}+x_{1}^{2} x_{3}\right) \\
= & 4 x_{1} x_{2} x_{4}+3 x_{1}^{3} x_{3}+2 x_{1}^{5}+x_{1} x_{3}^{2} .
\end{aligned}
$$

For $\mathcal{E}_{4}$ we have

$$
\begin{aligned}
\mathcal{E}_{4} & =\left(8 x_{4}+56 x_{1} x_{2}\right) \mathcal{B}_{3}+\left(9 x_{3}+36 x_{1}^{2}\right) \mathcal{C}_{3}+10(\cdots) \mathcal{D}_{3}+11 x_{1} \mathcal{E}_{3} \\
& =\left(3 x_{4}+x_{1} x_{2}\right)\left(2 x_{1}^{2} x_{2}\right)+\left(4 x_{3}+x_{1}^{2}\right)\left(x_{1}^{4}+x_{1}^{2} x_{3}\right)+x_{1}\left(4 x_{1} x_{2} x_{4}+3 x_{1}^{3} x_{3}+2 x_{1}^{5}+x_{1} x_{3}^{2}\right) \\
& =2 x_{1}^{3} x_{2}^{2}+3 x_{1}^{4} x_{3}+3 x_{1}^{6} .
\end{aligned}
$$

Finally $\mathcal{E}_{5}$

$$
\begin{aligned}
\mathcal{E}_{5} & =11 x_{3} \mathcal{C}_{4}+13 x_{1} \mathcal{E}_{4} \\
& =x_{3}\left(x_{1}^{2} x_{2}^{2}+4 x_{1}\left(x_{1}^{4}+x_{1}^{2} x_{3}\right)\right)+3 x_{1}\left(2 x_{1}^{3} x_{2}^{2}+3 x_{1}^{4} x_{3}+3 x_{1}^{6}\right) \\
& =4 x_{1}^{7}+x_{1}^{4} x_{2}^{2}+3 x_{1}^{5} x_{3}+x_{1}^{2} x_{2}^{2} x_{3}+4 x_{1}^{3} x_{3}^{2} \\
& =x_{1}^{3}\left(x_{1}+\frac{x_{3}}{x_{1}}\right)\left(4 x_{1}^{3}+x_{2}^{2}+4 x_{1} x_{3}\right) .
\end{aligned}
$$

Note that $-3 / 2 \equiv 1(\bmod 5)$ and thus $\left(4 x_{1}^{3}+x_{2}^{2}+4 x_{1} x_{3}\right)=\left(\frac{3}{2} x_{1}^{3}+x_{2}^{2}-x_{1} x_{3}\right)$. The proof is completed by specializing for each $i \in\{1,2,3\} x_{i}$ to $a_{i+1}$,

$$
\begin{gathered}
\widetilde{\mathcal{C}}_{5}\left(a_{2}, a_{3}, a_{4}\right)=\alpha_{1}, \\
\widetilde{\mathcal{D}}_{5}\left(a_{2}, a_{3}, a_{4}\right)=\frac{a_{3}}{a_{2}} \alpha_{1}=\beta_{1},
\end{gathered}
$$


and

$$
\widetilde{\mathcal{E}}_{5}\left(a_{2}, a_{3}, a_{4}\right)=\left(a_{2}+\frac{a_{4}}{a_{2}}\right) \alpha_{1}=-\left(\frac{3 a_{2}}{2}-\frac{a_{4}}{a_{2}}\right) \alpha_{1}=\gamma_{1} .
$$

This completes the proof of the Lemma for the case $p=5$.

Case $3, p \geq 7$. Note for $p \geq 7$ all lemmas stated in $\S 3$ apply.

The equations (4.1), (4.2) and (4.3) were explicitly solved in [Nor17, page 267ff]:

$$
\begin{gathered}
\mathcal{A}_{m}=x_{1}^{m}(2 m-1) ! !, \\
\mathcal{B}_{m}=x_{1}^{m-1} x_{2} \mathcal{R}_{m},
\end{gathered}
$$

and

$$
\mathcal{C}_{m}=x_{1}^{m+1} \mathcal{S}_{m}(1,-1)+x_{1}^{m-1} x_{3} \mathcal{S}_{m}(0,1)+x_{1}^{m-2} x_{2}^{2}\left(\mathcal{S}_{m}(2,0)-\mathcal{T}_{m}\right) .
$$

The remaining part of the proof is to explicitly calculate $\mathcal{D}_{m}$ and $\mathcal{E}_{m}$ and compute the corresponding reductions $\widetilde{\mathcal{D}}_{p}$ and $\widetilde{\mathcal{E}}_{p}$. Insertion of (4.7), (4.8) and (4.9) into (4.4) yields

$$
\begin{aligned}
\mathcal{D}_{m+1} & =\left(x_{4}+x_{1} x_{2}(2 m)\right) x_{1}^{m}(2 m+1) ! ! \\
& +x_{1}^{m-1} x_{2} \mathcal{R}_{m}\left(x_{3}(2 m+2)+x_{1}^{2}(m+1)(2 m+1)\right) \\
& +x_{2}(2 m+3)\left(x_{1}^{m+1} \mathcal{S}_{m}(1,-1)+x_{1} x_{3} \mathcal{S}_{m}(0,1)+x_{1}^{m-2} x_{2}^{2}\left(\mathcal{S}_{m}(2,0)-\mathcal{T}_{m}\right)\right) \\
& +x_{1}(2 m+4) \mathcal{D}_{m} .
\end{aligned}
$$

Put $\phi_{m}:=x_{4}(2 m+2) !$ !. Then $\widetilde{\phi}_{p}=0$. Lemma 14 yields

$$
\begin{aligned}
\mathcal{D}_{m} & =\phi_{m}+x_{1}^{m-3}(2 m+2) ! ! \sum_{j=1}^{m-1} \frac{1}{(2 j+4) ! !}\left(\left(x_{1}^{2} x_{4}+x_{1}^{3} x_{2}(2 j)\right)(2 j+1) ! !\right. \\
& +\left(x_{1}^{2}(j+1)(2 j+1)+x_{3}(2 j+2)\right) x_{1} x_{2} \mathcal{R}_{j} \\
& \left.+x_{2}(2 j+3)\left(x_{1}^{3} \mathcal{S}_{j}(1,-1)+x_{1} x_{3} \mathcal{S}_{j}(0,1)+x_{2}^{2}\left(\mathcal{S}_{j}(2,0)-\mathcal{T}_{j}\right)\right)\right) .
\end{aligned}
$$

In view of the definitions in Lemma 10 we have

$$
\begin{aligned}
\mathcal{D}_{m}= & \phi_{m}+x_{1}^{m} x_{2}\left(\mathcal{X}_{m}(2,0)+\mathcal{U}_{m}(1,-1)+\mathcal{V}_{m}(2,3,1)\right) \\
& +x_{1}^{m-3} x_{2}^{3}\left(\mathcal{U}_{m}(2,0)-\mathcal{W}_{m}\right) \\
& +x_{1}^{m-1} x_{4} \mathcal{X}_{m}(1,0) \\
& +x_{1}^{m-2} x_{2} x_{3}\left(\mathcal{U}_{m}(0,1)+\mathcal{V}_{m}(0,2,2)\right)
\end{aligned}
$$

Inserting the explicit solution for $\mathcal{D}_{m}$ in (4.5) yields

$$
\begin{aligned}
\mathcal{E}_{m+1} & =x_{1}(2 m+5) \mathcal{E}_{m} \\
& +x_{1}^{m}(2 m-1) ! !\left(x_{5}(2 m+1)+x_{1} x_{3}(2 m+1)(2 m)+x_{1}^{3}\left(\begin{array}{c}
2 m+1 \\
3
\end{array}\right)+x_{2}^{2}\left(\begin{array}{c}
2 m+1 \\
2
\end{array}\right)\right) \\
& +x_{1}^{m-1} x_{2} \mathcal{R}_{m}\left(x_{1} x_{2}(2 m+2)(2 m+1)+x_{4}(2 m+2)\right) \\
& +x_{1}^{m-2}(2 m+3)\left(x_{3}+x_{1}^{2}(m+1)\right)\left(x_{1}^{3} \mathcal{S}_{m}(1,-1)+x_{1} x_{3} \mathcal{S}_{m}(0,1)+x_{2}^{2}\left(\mathcal{S}_{m}(2,0)-\mathcal{T}_{m}\right)\right) \\
& +x_{2}(2 m+4)\left(x_{1}^{m} x_{2}\left(\mathcal{X}_{m}(2,0)+\mathcal{U}_{m}(1,-1)+\mathcal{V}_{m}(2,3,1)\right)\right. \\
& +x_{1}^{m-3} x_{2}^{3}\left(\mathcal{U}_{m}(2,0)-\mathcal{W}_{m}\right)+x_{1}^{m-1} x_{4} \mathcal{X}_{m}(1,0) \\
& \left.+x_{1}^{m-2} x_{2} x_{3}\left(\mathcal{U}_{m}(0,1)+\mathcal{V}_{m}(0,2,2)\right)\right) .
\end{aligned}
$$


Put $\phi_{m}^{\prime}:=x_{5}(2 m+3) !$ !. Then $\widetilde{\phi}_{p}^{\prime}=0$. Lemma 14 yields

$$
\begin{aligned}
\mathcal{E}_{m}= & \phi_{m}^{\prime}+x_{1}^{m+2}(2 m+3) ! ! \sum_{j=1}^{m-1} \frac{j(2 j-1)}{3(2 j+3)(2 j+5)}+\frac{(j+1)(2 j+3)}{(2 j+5) ! !} \mathcal{S}_{j}(1,-1) \\
& +x_{1}^{m-1} x_{2}^{2}(2 m+3) ! ! \sum_{j=1}^{m-1}\left(\frac{j(2 j+1) ! !}{(2 j+5) ! !}+\frac{(j+1)(2 j+3)}{(2 j+5) ! !}\left(\mathcal{S}_{j}(2,0)-\mathcal{T}_{j}\right)\right. \\
& \left.+\frac{(j+1)(2 j+1)}{(2 j+5) ! !} \mathcal{R}_{j}+\frac{(2 j+4)}{(2 j+5) ! !}\left(\mathcal{X}_{j}(2,0)+\mathcal{V}_{j}(2,3,1)+\mathcal{U}_{j}(1,-1)\right)\right) \\
& +x_{1}^{m-4} x_{2}^{4}(2 m+3) ! ! \sum_{j=1}^{m-1} \frac{(2 j+4)}{(2 j+5) ! !}\left(\mathcal{U}_{j}(2,0)-\mathcal{W}_{j}\right) \\
& +x_{1}^{m-3} x_{2}^{2} x_{3}(2 m+3) ! ! \sum_{j=1}^{m-1} \frac{(2 j+4)}{(2 j+5) ! !}\left(\mathcal{U}_{j}(0,1)+\mathcal{V}_{j}(0,2,2)\right)+\frac{2 j+3}{(2 j+5) ! !}\left(\mathcal{S}_{j}(2,0)-\mathcal{T}_{j}\right) \\
& +x_{1}^{m} x_{3}(2 m+3) ! ! \sum_{j=1}^{m-1} \frac{(j+1)(2 j+3)}{(2 j+5) ! !} \mathcal{S}_{j}(0,1)+\frac{2 j}{(2 j+3)(2 j+5)}+\frac{2 j}{(2 j+5) ! !} \mathcal{S}_{j}(1,-1) \\
& +x_{1}^{m-2} x_{2} x_{4}(2 m+3) ! ! \sum_{j=1}^{m-1} \frac{(2 j+4)}{(2 j+5) ! !} \mathcal{X}_{j}(1,0)+\frac{2 j+2}{(2 j+5) ! !} \mathcal{R}_{j} \\
& +x_{1}^{m-2} x_{3}^{2}(2 m+3) ! ! \sum_{j=1}^{m-1} \frac{2 j+3}{(2 j+5) ! !} \mathcal{S}_{j}(0,1) \\
& +x_{1}^{m-1} x_{5}(2 m+3) ! ! \sum_{j=1}^{m-1} \frac{1}{(2 j+3)(2 j+5)} .
\end{aligned}
$$

In view of the definitions in Lemma 12 and Lemma 13 we have

$$
\begin{aligned}
\mathcal{E}_{m}= & \phi_{m}^{\prime}+x_{1}^{m+2}\left(\mathcal{Z}_{m}(2 / 3,-1 / 3,0)+\widehat{\mathcal{S}}_{m}(1,-1,1)\right) \\
& +x_{1}^{m-1} x_{2}^{2}\left(\mathcal{Z}_{m}(0,1,0)+\widehat{\mathcal{S}}_{m}(2,0,1)-\widehat{\mathcal{T}}_{m}(1)+\frac{1}{2} \widehat{\mathcal{R}}_{m}(2)+\widehat{\mathcal{X}}_{m}(2,0)+\widehat{\mathcal{V}}_{m}(2,3,1)+\widehat{\mathcal{U}}_{m}(1,-1)\right) \\
& +x_{1}^{m-4} x_{2}^{4}\left(\widehat{\mathcal{U}}_{m}(2,0)-\widehat{\mathcal{W}}_{m}\right)+x_{1}^{m-3} x_{2}^{2} x_{3}\left(\widehat{\mathcal{U}}_{m}(0,1)+\widehat{\mathcal{V}}_{m}(0,2,2)+\widehat{\mathcal{S}}_{m}(2,0,0)-\widehat{\mathcal{T}}_{m}(0)\right) \\
& +x_{1}^{m} x_{3}\left(\widehat{\mathcal{S}}_{m}(0,1,1)+\widehat{\mathcal{Z}}_{m}(0,2,0)+\widehat{\mathcal{S}}_{m}(1,-1,0)\right)+x_{1}^{m-2} x_{2} x_{4}\left(\widehat{\mathcal{X}}_{m}(0,1)+\widehat{\mathcal{R}}_{m}(0)\right) \\
& +x_{1}^{m-2} x_{3}^{2} \widehat{\mathcal{S}}_{m}(0,1,0)+x_{1}^{m-1} x_{5} \mathcal{Z}(0,0,1)
\end{aligned}
$$

Recall that

$$
\widehat{f}^{p}(\zeta) \equiv \mathcal{A}_{p} \zeta^{2 p+1}+\mathcal{B}_{p} \zeta^{2 p+2}+\mathcal{C}_{p} \zeta^{2 p+3}+\mathcal{D}_{p} \zeta^{2 p+4}+\mathcal{E}_{p} \zeta^{2 p+5} \bmod \left\langle\zeta^{2 p+6}\right\rangle .
$$

It follows from (4.7), (4.8) and Lemma 4 that

$$
\widetilde{\mathcal{A}}_{p}=\widetilde{\mathcal{B}}_{p}=0
$$

and from (4.9), Lemma 4 and Lemma 5 we obtain

$$
\widetilde{\mathcal{C}}_{p}=a_{1}^{p-2}\left(3 / 2 a_{1}^{3}+a_{2}^{2}-a_{2} a_{4}\right)=\alpha_{1} .
$$


In view of Lemma 10 we have

$$
\begin{gathered}
\widetilde{\mathcal{V}}_{p}(2,3,1)=-3, \quad \widetilde{\mathcal{U}}_{p}(1,-1)=9 / 2, \quad \widetilde{\mathcal{X}}_{p}(2,0)=0, \quad \widetilde{\mathcal{U}}_{p}(2,0)=3, \quad \widetilde{\mathcal{W}}_{p}=2, \\
\tilde{\mathcal{U}}_{p}(0,1)=-3, \quad \widetilde{\mathcal{V}}_{p}(0,2,2)=2 \quad \text { and } \quad \widetilde{\mathcal{X}}_{p}(1,0)=0 .
\end{gathered}
$$

For each $i \geq 1$ we specialize $x_{i}$ to $a_{i+1}$, and by (4.10) we obtain

$$
\begin{aligned}
\widetilde{\mathcal{D}}_{p}\left(a_{2}, a_{3}\right)= & (9 / 2-3) a_{2}^{p} a_{3}+(3-2) a_{2}^{p-3} a_{3}^{3} \\
& +(-3+2) a_{2}^{p-2} a_{3} a_{4} \\
= & a_{2}^{p-3} a_{3}\left(3 / 2 a_{2}^{3}+a_{3}^{2}-a_{2} a_{4}\right) \\
= & \frac{a_{3}}{a_{2}} \widetilde{\mathcal{C}}_{p}=\frac{a_{3}}{a_{2}} \alpha_{1}=\beta_{1} .
\end{aligned}
$$

Concerning $\mathcal{E}_{p}$ we have by Lemma 12 and Lemma 13

$$
\begin{gathered}
\widetilde{\mathcal{Z}}_{p}(2 / 3,-1 / 3,0)=\frac{9}{2}, \quad \widetilde{\mathcal{Z}}_{p}(0,1,0)=-\frac{3}{2}, \\
\widetilde{\widehat{\mathcal{S}}}_{p}(1,-1,1)=-\frac{27}{4}, \quad \widetilde{\widehat{\mathcal{T}}}_{p}(1)=-9, \quad \widetilde{\widehat{\mathcal{S}}}_{p}(2,0,1)=-12, \\
\widetilde{\widehat{\mathcal{R}}}_{p}(2)=15, \quad \widetilde{\widehat{\mathcal{X}}}_{p}(2,0)=-6, \quad \widetilde{\widehat{\mathcal{V}}}_{p}(2,3,1)=-\frac{33}{2}, \\
\widetilde{\widehat{\mathcal{U}}}_{p}(1,-1)=\frac{21}{2}, \quad \widetilde{\widehat{\mathcal{U}}}_{p}(2,0)=2, \quad \widetilde{\widehat{\mathcal{W}}}_{p}=2, \\
\widetilde{\widehat{\mathcal{U}}}_{p}(0,1)=-\frac{19}{2}, \quad \widetilde{\widehat{\mathcal{V}}}_{p}(0,2,2)=8, \quad \widetilde{\widehat{\mathcal{S}}}_{p}(0,1,1)=\frac{3}{4}, \quad \widetilde{\widehat{\mathcal{Z}}}_{p}(0,2,0)=-3 \\
\widetilde{\widehat{\mathcal{S}}}_{p}(1,-1,0)=\frac{21}{4}, \quad \widetilde{\widehat{\mathcal{S}}}_{p}(0,1,0)=-1, \quad \widetilde{\widehat{\mathcal{S}}}_{p}(2,0,0)=\frac{17}{2}, \quad \widetilde{\widehat{\mathcal{T}}}_{p}(0)=6
\end{gathered}
$$

and

$$
\widetilde{\widehat{\mathcal{X}}}_{p}(0,1)=3, \quad \widetilde{\widehat{\mathcal{R}}}_{p}(0)=-3, \quad \widetilde{\widehat{\mathcal{Z}}}_{p}(0,0,1)=0 .
$$

respectively. Hence, from (4.12) we obtain

$$
\begin{aligned}
\widetilde{\mathcal{E}}_{p}\left(a_{2}, a_{3}\right)= & \left(\frac{9}{2}-\frac{27}{4}\right) a_{2}^{p+2}+\left(-\frac{3}{2}-12+9+\frac{15}{2}-6-\frac{33}{2}+\frac{21}{2}\right) a_{2}^{p-1} a_{3}^{2} \\
& +(2-2) a_{2}^{p-4} a_{3}^{4}+\left(-\frac{19}{2}+8+\frac{17}{2}-6\right) a_{2}^{p-3} a_{3}^{2} a_{4} \\
& +\left(\frac{3}{4}-3+\frac{21}{4}\right) a_{2}^{p} a_{4}-a_{2}^{p-2} a_{4}^{2}+(3-3) a_{2}^{p-2} a_{3} a_{5} \\
= & -9 / 4 a_{2}^{p+2}-3 / 2 a_{2}^{p-1} a_{3}^{2}+a_{2}^{p-3} a_{3}^{2} a_{4}+3 a_{2}^{p} a_{4}-a_{2}^{p-2} a_{4}^{2} \\
= & -\left(\frac{3 a_{2}}{2}-\frac{a_{4}}{a_{2}}\right) \widetilde{\mathcal{C}}_{p}=-\left(\frac{3 a_{2}}{2}-\frac{a_{4}}{a_{2}}\right) \alpha_{1}=\gamma_{1} .
\end{aligned}
$$

This completes the proof of the Main Lemma.

Proof of Theorem B. As for the case of the proof of the Main Lemma we divide this proof into three cases, thus treating the special cases $p=3$ and $p=5$ separately.

By the Main Lemma the theorem is valid for $n=1$. Assume that the theorem is valid for some integer $n \geq 1$. Let $h(\zeta)=f^{p^{n}}(\zeta)$. Then,

$$
h(\zeta)-\zeta=\alpha_{n} \zeta^{2 d+1}+\beta_{n} \zeta^{2 d+2}+\gamma_{n} \zeta^{2 d+3}+\left\langle\zeta^{2 d+4}\right\rangle .
$$

For each integer $m \geq 1$ define the power series $\Delta_{m}(\zeta)$ inductively by $\Delta_{1}(\zeta):=h(\zeta)-\zeta$, and for $m \geq 2$ by

$$
\Delta_{m}(\zeta):=\Delta_{m-1}(h(\zeta))-\Delta_{m-1}(\zeta)
$$


Note that $\Delta_{p}(\zeta)=h^{p}(\zeta)-\zeta=f^{p^{n+1}}(\zeta)-\zeta$.

As in the proof of the Main Lemma we define $F_{\ell}:=\mathbb{Q}_{p}\left[x_{1}, x_{2}, \ldots, x_{\ell}\right]$, and $F_{\infty}:=\mathbb{Q}_{p}\left[x_{1}, x_{2}, \ldots\right]$. We recall that $d=\frac{p^{n}-1}{p-1}$ and put

$$
\widehat{\varphi}=\frac{3}{2} x_{1}^{3}+x_{2}^{2}-x_{1} x_{3}, \quad \widehat{\alpha}_{n}=\widehat{\alpha}_{n}(x):=x_{1}^{p^{n}-2 d} \widehat{\varphi}^{d},
$$

and

$$
\widehat{\beta}_{n}=\widehat{\beta}_{n}(x):=\frac{x_{2}}{x_{1}} \widehat{\alpha}_{n}(x), \quad \widehat{\gamma}_{n}=\widehat{\gamma}_{n}(x):=-\left(\frac{3 x_{1}}{2}-x_{3} x_{1}\right) \widehat{\alpha}_{n} .
$$

For future reference we note that

$$
\widehat{\beta}_{n}^{2}-\widehat{\alpha}_{n} \widehat{\gamma}_{n}=x_{1}^{-2} \widehat{\alpha}_{n}^{2} \widehat{\varphi} .
$$

Moreover we consider the power series $\widehat{h} \in F_{\infty}[[\zeta]]$ defined as

$$
\widehat{h}(\zeta)-\zeta:=\widehat{\alpha}_{n} \zeta^{2 d+1}+\widehat{\beta}_{n} \zeta^{2 d+2}+\widehat{\gamma}_{n} \zeta^{2 d+3}+\left\langle\zeta^{2 d+4}\right\rangle .
$$

For $m=1$ we define the relation $\widehat{\Delta}_{1}(\zeta):=\widehat{h}(\zeta)-\zeta$ and for each integer $m \geq 2$

$$
\widehat{\Delta}_{m}(\zeta):=\widehat{\Delta}_{m-1}(\widehat{h}(\zeta))-\widehat{\Delta}_{m-1}(\zeta) \text {. }
$$

We will prove that the first significant terms of $\widetilde{\Delta}_{p}(\zeta)$ are $\alpha_{n+1}, \beta_{n+1}$ and $\gamma_{n+1}$.

As for the proof of the Main Lemma in the cases of $p=3$ and $p=5$ we redefine the ground field of $F_{\ell}$ to be $\mathbb{F}_{p}$ for $p=3$ and $p=5$ respectively, and utilize, without comment, that the field characteristic is 3 and 5 .

Case 1, $p=3$. Let $\widehat{\delta}_{n}$ and $\widehat{\varepsilon}_{n}$ denote the coefficients of the terms of order $2 d+4$ and $2 d+5$ in $\widehat{h}$ respectively. By definition $d \equiv 1(\bmod p)$. We obtain

$$
\begin{aligned}
\widehat{\Delta}_{2}(\zeta) \equiv & \widehat{\Delta}_{1}(\widehat{h}(\zeta))-\widehat{\Delta}_{1}(\zeta) \\
\equiv & \widehat{\alpha}_{n}\left[\left(\zeta+\widehat{\Delta}_{1}\right)^{2 d+1}-\zeta^{2 d+1}\right]+\widehat{\beta}_{n}\left[\left(\zeta+\widehat{\Delta}_{1}\right)^{2 d+2}-\zeta^{2 d+2}\right] \\
& +\widehat{\gamma}_{n}\left[\left(\zeta+\widehat{\Delta}_{1}\right)^{2 d+3}-\zeta^{2 d+3}\right]+\widehat{\delta}_{n}\left[\left(\zeta+\widehat{\Delta}_{1}\right)^{2 d+4}-\zeta^{2 d+4}\right] \\
& +\widehat{\varepsilon}_{n}\left[\left(\zeta+\widehat{\Delta}_{1}\right)^{2 d+5}-\zeta^{2 d+5}\right] \\
\equiv & 3 \widehat{\alpha}_{n}(\ldots)+4 \widehat{\alpha}_{n} \widehat{\beta}_{n} \zeta^{4 d+2}+4 \widehat{\beta}_{n}^{2} \zeta^{4 d+3}+4 \widehat{\beta}_{n} \widehat{\gamma}_{n} \zeta^{4 d+4}+4 \widehat{\beta}_{n} \widehat{\delta}_{n} \zeta^{4 d+5} \\
& +5 \widehat{\alpha}_{n} \widehat{\gamma}_{n} \zeta^{4 d+3}+5 \widehat{\beta}_{n} \widehat{\gamma}_{n} \zeta^{4 d+4}+5 \widehat{\gamma}_{n}^{2} \zeta^{4 d+5}+6 \widehat{\delta}_{n}(\ldots)+7 \widehat{\alpha}_{n} \widehat{\varepsilon}_{n} \zeta^{4 d+5} \\
\equiv & \widehat{\alpha}_{n} \widehat{\beta}_{n} \zeta^{4 d+2}+\left(\widehat{\beta}_{n}^{2}+2 \widehat{\alpha}_{n} \widehat{\gamma}_{n}\right) \zeta^{4 d+3}+\left(\widehat{\alpha}_{n} \widehat{\varepsilon}_{n}+2 \widehat{\gamma}_{n}^{2}+\widehat{\beta}_{n} \widehat{\delta}_{n}\right) \zeta^{4 d+5} \bmod \left\langle\zeta^{4 d+6}\right\rangle .
\end{aligned}
$$

Also,

$$
\begin{aligned}
\widehat{\Delta}_{3}(\zeta) \equiv & \widehat{\Delta}_{2}\left(\widehat{h}_{h}(\zeta)\right)-\widehat{\Delta}_{2}(\zeta) \\
\equiv & \widehat{\alpha}_{n} \widehat{\beta}_{n}\left[\left(\zeta+\widehat{\Delta}_{1}\right)^{4 d+2}-\zeta^{4 d+2}\right]+\left(\widehat{\beta}_{n}^{2}+2 \widehat{\alpha}_{n} \widehat{\gamma}_{n}\right)\left[\left(\zeta+\widehat{\Delta}_{1}\right)^{4 d+3}-\zeta^{4 d+3}\right] \\
& +\left(\widehat{\alpha}_{n} \widehat{\varepsilon}_{n}+2 \widehat{\gamma}_{n}^{2}+\widehat{\beta}_{n} \widehat{\delta}_{n}\right)\left[\left(\zeta+\widehat{\Delta}_{1}\right)^{4 d+5}-\zeta^{4 d+5}\right] \\
6 \widehat{\alpha}_{n} \widehat{\beta}_{n}(\ldots)+7 \widehat{\alpha}_{n}\left(\widehat{\beta}_{n}^{2}+2 \widehat{\alpha}_{n} \widehat{\gamma}_{n}\right) \zeta^{6 d+3}+7 \widehat{\beta}_{n}\left(\widehat{\beta}_{n}^{2}+2 \widehat{\alpha}_{n} \widehat{\gamma}_{n}\right) \zeta^{6 d+4} & \\
& +7 \widehat{\gamma}_{n}\left(\widehat{\beta}_{n}^{2}+2 \widehat{\alpha}_{n} \widehat{\gamma}_{n}\right) \zeta^{6 d+5}+9\left(\widehat{\alpha}_{n} \widehat{\varepsilon}_{n}+2 \widehat{\gamma}_{n}^{2}+\widehat{\beta}_{n} \widehat{\delta}_{n}\right)(\ldots) \\
\equiv & \widehat{\alpha}_{n}\left(\widehat{\beta}_{n}^{2}-\widehat{\alpha}_{n} \widehat{\gamma}_{n}\right) \zeta^{6 d+3}+\widehat{\beta}_{n}\left(\widehat{\beta}_{n}^{2}-\widehat{\alpha}_{n} \widehat{\gamma}_{n}\right) \zeta^{6 d+4}+\widehat{\gamma}_{n}\left(\widehat{\beta}_{n}^{2}-\widehat{\alpha}_{n} \widehat{\gamma}_{n}\right) \zeta^{6 d+5} \bmod \left\langle\zeta^{6 d+6}\right\rangle .
\end{aligned}
$$

Recall that $d=\frac{p^{n}-1}{p-1}$ by which we obtain $d p+1=\frac{p^{n+1}-1}{p-1}$ and thus we see that

$\widehat{\Delta}_{3}(\zeta) \equiv \widehat{\alpha}_{n}\left(\widehat{\beta}_{n}^{2}-\widehat{\alpha}_{n} \widehat{\gamma}_{n}\right) \zeta^{2(3 d+1)+1}+\widehat{\beta}_{n}\left(\widehat{\beta}_{n}^{2}-\widehat{\alpha}_{n} \widehat{\gamma}_{n}\right) \zeta^{2(3 d+1)+2}+\widehat{\gamma}_{n}\left(\widehat{\beta}_{n}^{2}-\widehat{\alpha}_{n} \widehat{\gamma}_{n}\right) \zeta^{2(3 d+1)+3} \bmod \left\langle\zeta^{2(3 d+1)+4}\right\rangle$. 
In view of (4.18) we have

$$
\begin{gathered}
\widehat{\alpha}_{n}\left(\widehat{\beta}_{n}^{2}-\widehat{\alpha}_{n} \widehat{\gamma}_{n}\right)=\widehat{\alpha}_{n}^{3} x_{1}^{-2} \widehat{\varphi}=x_{1}^{-2}\left(x_{1}^{3^{n}-2 d} \widehat{\varphi}^{d}\right)^{3} \widehat{\varphi}=x_{1}^{3^{n+1}-2(3 d+1)} \widehat{\varphi}^{3 d+1}=\widehat{\alpha}_{n+1} \\
\widehat{\beta}_{n}\left(\widehat{\beta}_{n}^{2}-\widehat{\alpha}_{n} \widehat{\gamma}_{n}\right)=\frac{x_{2}}{x_{1}} \widehat{\alpha}_{n+1}=\widehat{\beta}_{n+1},
\end{gathered}
$$

and

$$
\widehat{\gamma}_{n}\left(\widehat{\beta}_{n}^{2}-\widehat{\alpha}_{n} \widehat{\gamma}_{n}\right)=\frac{x_{3}}{x_{1}} \widehat{\alpha}_{n+1}=\widehat{\gamma}_{n+1}
$$

The proof is finished by specializing for each $i \in\{1,2,3\} x_{i}$ to $a_{i+1}$ from which we obtain

$\widetilde{\Delta}_{3}(\zeta)=f^{3^{n+1}}(\zeta)-\zeta=\alpha_{n+1} \zeta^{2(3 d+1)+1}+\beta_{n+1} \zeta^{2(3 d+1)+2}+\gamma_{n+1} \zeta^{2(3 d+1)+3} \bmod \left\langle\zeta^{2(3 d+1)+4}\right\rangle$

This completes the induction and thus the proof of the Theorem for the case $p=3$.

Case 2, $p=5$. As for the case of $p=3$ we let $\widehat{\delta}_{n}$ and $\widehat{\varepsilon}_{n}$ denote the coefficients of the terms of order $2 d+4$ and $2 d+5$ in $\widehat{h}$ respectively. Recall that $d \equiv 1(\bmod p)$. We obtain

$$
\begin{aligned}
& \widehat{\Delta}_{2}(\zeta) \equiv \widehat{\Delta}_{1}(\widehat{h}(\zeta))-\widehat{\Delta}_{1}(\zeta) \\
& \equiv \widehat{\alpha}_{n}\left[\left(\zeta+\widehat{\Delta}_{1}\right)^{2 d+1}-\zeta^{2 d+1}\right]+\widehat{\beta}_{n}\left[\left(\zeta+\widehat{\Delta}_{1}\right)^{2 d+2}-\zeta^{2 d+2}\right] \\
&+\widehat{\gamma}_{n}\left[\left(\zeta+\widehat{\Delta}_{1}\right)^{2 d+3}-\zeta^{2 d+3}\right]+\widehat{\delta}_{n}\left[\left(\zeta+\widehat{\Delta}_{1}\right)^{2 d+4}-\zeta^{2 d+4}\right] \\
&+\widehat{\varepsilon}_{n}\left[\left(\zeta+\widehat{\Delta}_{1}\right)^{2 d+5}-\zeta^{2 d+5}\right] \\
& \equiv 3 \widehat{\alpha}_{n}^{2} \zeta^{4 d+1}+3 \widehat{\alpha}_{n} \widehat{\beta}_{n} \zeta^{4 d+2}+3 \widehat{\alpha}_{n} \widehat{\gamma}_{n} \zeta^{4 d+3}+3 \widehat{\alpha}_{n} \widehat{\delta}_{n} \zeta^{4 d+4}+3 \widehat{\alpha}_{n} \widehat{\varepsilon}_{n} \zeta^{4 d+5} \\
&+4 \widehat{\alpha}_{n} \widehat{\beta}_{n} \zeta^{4 d+2}+4 \widehat{\beta}_{n}^{2} \zeta^{4 d+3}+4 \widehat{\beta}_{n} \widehat{\gamma}_{n} \zeta^{4 d+4}+4 \widehat{\beta}_{n} \widehat{\delta}_{n} \zeta^{4 d+5} \\
&+5 \widehat{\gamma}_{n}(\ldots)+6 \widehat{\alpha}_{n} \widehat{\delta}_{n} \zeta^{4 d+4}+6 \widehat{\beta}_{n} \widehat{\delta}_{n} \zeta^{4 d+5}+7 \widehat{\alpha}_{n} \widehat{\varepsilon}_{n} \zeta^{4 d+5} \\
& \equiv 3 \widehat{\alpha}_{n}^{2} \zeta^{4 d+1}+2 \widehat{\alpha}_{n} \widehat{\beta}_{n} \zeta^{4 d+2}+\left(4 \widehat{\beta}_{n}^{2}+3 \widehat{\alpha}_{n} \widehat{\gamma}_{n}\right) \zeta^{4 d+3} \\
&+4\left(\widehat{\alpha}_{n} \widehat{\delta}_{n}+\widehat{\beta}_{n} \widehat{\gamma}_{n}\right) \zeta^{4 d+4} \bmod \left\langle\zeta^{4 d+6}\right\rangle .
\end{aligned}
$$

Continuing for $\widehat{\Delta}_{3}$ we have

$$
\begin{aligned}
\widehat{\Delta}_{3}(\zeta) \equiv & \widehat{\Delta}_{2}(\widehat{h}(\zeta))-\widehat{\Delta}_{2}(\zeta) \\
\equiv & 3 \widehat{\alpha}_{n}^{2}\left[\left(\zeta+\widehat{\Delta}_{1}\right)^{4 d+1}-\zeta^{4 d+1}\right]+2 \widehat{\alpha}_{n} \widehat{\beta}_{n}\left[\left(\zeta+\widehat{\Delta}_{1}\right)^{4 d+2}-\zeta^{4 d+2}\right] \\
& +\left(4 \widehat{\beta}_{n}^{2}+3 \widehat{\alpha}_{n} \widehat{\gamma}_{n}\right)\left[\left(\zeta+\widehat{\Delta}_{1}\right)^{4 d+3}-\zeta^{4 d+3}\right]+\left(\widehat{\alpha}_{n} \widehat{\delta}_{n}+4 \widehat{\beta}_{n} \widehat{\gamma}_{n}\right)\left[\left(\zeta+\widehat{\Delta}_{1}\right)^{4 d+4}-\zeta^{4 d+4}\right] \\
\equiv & 15 \widehat{\alpha}_{n}^{2}(\ldots)+12 \widehat{\alpha}_{n}^{2} \widehat{\beta}_{n} \zeta^{6 d+2}+12 \widehat{\alpha}_{n} \widehat{\beta}_{n}^{2} \zeta^{6 d+3}+12 \widehat{\alpha}_{n} \widehat{\beta}_{n} \widehat{\gamma}_{n} \zeta^{6 d+4}+12 \widehat{\alpha}_{n} \widehat{\beta}_{n} \widehat{\delta}_{n} \zeta^{6 d+5} \\
& +7 \widehat{\alpha}_{n}\left(4 \widehat{\beta}_{n}^{2}+3 \widehat{\alpha}_{n} \widehat{\gamma}_{n}\right) \zeta^{6 d+3}+7 \widehat{\beta}_{n}\left(4 \widehat{\beta}_{n}^{2}+3 \widehat{\alpha}_{n} \widehat{\gamma}_{n}\right) \zeta^{6 d+4}+7 \widehat{\gamma}_{n}\left(4 \widehat{\beta}_{n}^{2}+3 \widehat{\alpha}_{n} \widehat{\gamma}_{n}\right) \zeta^{6 d+5} \\
& +32 \widehat{\alpha}_{n}\left(\widehat{\alpha}_{n} \widehat{\delta}_{n}+\widehat{\beta}_{n} \widehat{\gamma}_{n}\right) \zeta^{6 d+4}+32 \widehat{\beta}_{n}\left(\widehat{\alpha}_{n} \widehat{\delta}_{n}+\widehat{\beta}_{n} \widehat{\gamma}_{n}\right) \zeta^{6 d+5} \\
\equiv & 2 \widehat{\alpha}_{n}^{2} \widehat{\beta}_{n} \zeta^{6 d+2}+\widehat{\alpha}_{n}^{2} \widehat{\gamma}_{n} \zeta^{6 d+3}+\left(3 \widehat{\beta}_{n}^{3}+2 \widehat{\alpha}_{n}^{2} \widehat{\delta}_{n}\right) \zeta^{6 d+4} \\
& +\left(\widehat{\alpha}_{n} \widehat{\gamma}_{n}^{2}+4 \widehat{\alpha}_{n} \widehat{\beta}_{n} \widehat{\delta}_{n}\right) \zeta^{6 d+5} \bmod \left\langle\zeta^{6 d+6}\right\rangle .
\end{aligned}
$$


For $\widehat{\Delta}_{4}$ we have

$$
\begin{aligned}
\widehat{\Delta}_{4}(\zeta) \equiv & \widehat{\Delta}_{3}(\widehat{h}(\zeta))-\widehat{\Delta}_{3}(\zeta) \\
\equiv & 2 \widehat{\alpha}_{n}^{2} \widehat{\beta}_{n}\left[\left(\zeta+\widehat{\Delta}_{1}\right)^{6 d+2}-\zeta^{6 d+2}\right]+\widehat{\alpha}_{n}^{2} \widehat{\gamma}_{n}\left[\left(\zeta+\widehat{\Delta}_{1}\right)^{6 d+3}-\zeta^{6 d+3}\right] \\
& +\left(3 \widehat{\beta}_{n}^{3}+2 \widehat{\alpha}_{n}^{2} \widehat{\delta}_{n}\right)\left[\left(\zeta+\widehat{\Delta}_{1}\right)^{6 d+4}-\zeta^{6 d+4}\right]+\left(\widehat{\alpha}_{n} \widehat{\gamma}_{n}^{2}+4 \widehat{\alpha}_{n} \widehat{\beta}_{n} \widehat{\delta}_{n}\right)\left[\left(\zeta+\widehat{\Delta}_{1}\right)^{6 d+5}-\zeta^{6 d+5}\right] \\
\equiv & 16 \widehat{\alpha}_{n}^{3} \widehat{\beta}_{n} \zeta^{8 d+2}+16 \widehat{\alpha}_{n}^{2} \widehat{\beta}_{n}^{2} \zeta^{8 d+3}+16 \widehat{\alpha}_{n}^{2} \widehat{\beta}_{n} \widehat{\gamma}_{n} \zeta^{8 d+4}+16 \widehat{\alpha}_{n}^{2} \widehat{\beta}_{n} \widehat{\delta}_{n} \zeta^{8 d+5} \\
& +9 \widehat{\alpha}_{n}^{3} \widehat{\gamma}_{n} \zeta^{8 d+3}+9 \widehat{\alpha}_{n}^{2} \widehat{\beta}_{n} \widehat{\gamma}_{n} \zeta^{8 d+4}+9 \widehat{\alpha}_{n}^{2} \widehat{\gamma}_{n}^{2} \zeta^{8 d+5} \\
& +10\left(3 \widehat{\beta}_{n}^{3}+2 \widehat{\alpha}_{n}^{2} \widehat{\delta}_{n}\right)(\ldots)+11 \widehat{\alpha}_{n}\left(\widehat{\alpha}_{n} \widehat{\gamma}_{n}^{2}+4 \widehat{\alpha}_{n} \widehat{\beta}_{n} \widehat{\delta}_{n}\right) \zeta^{8 d+5} \\
\equiv & \widehat{\alpha}_{n}^{3} \widehat{\beta}_{n} \zeta^{8 d+2}+\widehat{\alpha}_{n}^{2}\left(\widehat{\beta}_{n}^{2}+4 \widehat{\alpha}_{n} \widehat{\gamma}_{n}\right) \zeta^{8 d+3} \bmod \left\langle\zeta^{8 d+6}\right\rangle .
\end{aligned}
$$

Finally for $\widehat{\Delta}_{5}$ we have

$$
\begin{aligned}
\widehat{\Delta}_{5}(\zeta) \equiv & \widehat{\Delta}_{4}(\widehat{h}(\zeta))-\widehat{\Delta}_{4}(\zeta) \\
\equiv & \widehat{\alpha}_{n}^{3} \widehat{\beta}_{n}\left[\left(\zeta+\widehat{\Delta}_{1}\right)^{8 d+2}-\zeta^{8 d+2}\right]+\widehat{\alpha}_{n}^{2}\left(\widehat{\beta}_{n}^{2}+4 \widehat{\alpha}_{n} \widehat{\gamma}_{n}\right)\left[\left(\zeta+\widehat{\Delta}_{1}\right)^{8 d+3}-\zeta^{8 d+3}\right] \\
\equiv & 10 \widehat{\alpha}_{n}^{3} \widehat{\beta}_{n}(\ldots)+11 \widehat{\alpha}_{n}^{3}\left(\widehat{\beta}_{n}^{2}+4 \widehat{\alpha}_{n} \widehat{\gamma}_{n}\right) \zeta^{10 d+3}+11 \widehat{\alpha}_{n}^{2} \widehat{\beta}_{n}\left(\widehat{\beta}_{n}^{2}+4 \widehat{\alpha}_{n} \widehat{\gamma}_{n}\right) \zeta^{10 d+4} \\
& +11 \widehat{\alpha}_{n}^{2} \widehat{\gamma}_{n}\left(\widehat{\beta}_{n}^{2}+4 \widehat{\alpha}_{n} \widehat{\gamma}_{n}\right) \zeta^{10 d+5} \\
\equiv & \widehat{\alpha}_{n}^{3}\left(\widehat{\beta}_{n}^{2}-\widehat{\alpha}_{n} \widehat{\gamma}_{n}\right) \zeta^{10 d+3}+\widehat{\alpha}_{n}^{2} \widehat{\beta}_{n}\left(\widehat{\beta}_{n}^{2}-\widehat{\alpha}_{n} \widehat{\gamma}_{n}\right) \zeta^{10 d+4} \\
& +\widehat{\alpha}_{n}^{2} \widehat{\gamma}_{n}\left(\widehat{\beta}_{n}^{2}-\widehat{\alpha}_{n} \widehat{\gamma}_{n}\right) \zeta^{10 d+5} \bmod \left\langle\zeta^{10 d+6}\right\rangle .
\end{aligned}
$$

In view of (4.18) we have

$$
\begin{gathered}
\widehat{\alpha}_{n}^{3}\left(\widehat{\beta}_{n}^{2}-\widehat{\alpha}_{n} \widehat{\gamma}_{n}\right)=\widehat{\alpha}_{n}^{5} x_{1}^{-2} \widehat{\varphi}=x_{1}^{-2}\left(x_{1}^{5^{n}-2 d} \widehat{\varphi}^{d}\right)^{5} \widehat{\varphi}=x_{1}^{5^{n+1}-2(5 d+1)} \widehat{\varphi}^{5 d+1}=\widehat{\alpha}_{n+1}, \\
\widehat{\alpha}_{n}^{2} \widehat{\beta}_{n}\left(\widehat{\beta}_{n}^{2}-\widehat{\alpha}_{n} \widehat{\gamma}_{n}\right)=\frac{x_{2}}{x_{1}} \widehat{\alpha}_{n+1}=\widehat{\beta}_{n+1},
\end{gathered}
$$

and finally

$$
\widehat{\alpha}_{n}^{2} \widehat{\gamma}_{n}\left(\widehat{\beta}_{n}^{2}-\widehat{\alpha}_{n} \widehat{\gamma}_{n}\right)=-\left(\frac{3 x_{1}}{2}-\frac{x_{3}}{x_{1}}\right) \widehat{\alpha}_{n+1}=\widehat{\gamma}_{n+1} .
$$

The proof is completed by specializing for each $i \in[1,3] x_{i}$ to $a_{i+1}$, which yields

$$
\widetilde{\Delta}_{5}(\zeta)=f^{5^{n+1}}(\zeta)-\zeta=\alpha_{n+1} \zeta^{2(5 d+1)+1}+\beta_{n+1} \zeta^{2(5 d+1)+2}+\gamma_{n+1} \zeta^{2(5 d+1)+3} \bmod \left\langle\zeta^{2(5 d+1)+4}\right\rangle .
$$

Case 3, $p \geq 7$. We recall that

$$
\widehat{\varphi}=\frac{3}{2} x_{1}^{3}+x_{2}^{2}-x_{1} x_{3}, \quad \widehat{\alpha}_{n}=\widehat{\alpha}_{n}(x):=x_{1}^{p^{n}-2 d} \widehat{\varphi}^{d},
$$

and

$$
\widehat{\beta}_{n}=\widehat{\beta}_{n}(x):=\frac{x_{2}}{x_{1}} \widehat{\alpha}_{n}(x), \quad \widehat{\gamma}_{n}=\widehat{\gamma}_{n}(x):=-\left(\frac{3 x_{1}}{2}-x_{3} x_{1}\right) \widehat{\alpha}_{n}
$$

Also let $\widehat{\delta}_{n}, \widehat{\varepsilon}_{n}$ be the coefficients of the terms of degree $2 d+4$ and $2 d+5$ in $\widehat{h}$ respectively, and $x=\left(x_{1}, x_{2}, \ldots\right)$. We will prove that for a given $m \geq 1$ we have

(4.19) $\widehat{\Delta}_{m}(\zeta) \equiv \widehat{\mathcal{A}}_{m} \zeta^{2 d m+1}+\widehat{\mathcal{B}}_{m} \zeta^{2 d m+2}+\widehat{\mathcal{C}}_{m} \zeta^{2 d m+3}+\widehat{\mathcal{D}}_{m} \zeta^{2 d m+4}+\widehat{\mathcal{E}}_{m} \zeta^{2 d m+5} \bmod \left\langle\zeta^{2 d m+6}\right\rangle$. 
where $\widehat{\mathcal{A}}=\widehat{\mathcal{A}}(x), \widehat{\mathcal{B}}=\widehat{\mathcal{B}}(x), \widehat{\mathcal{C}}=\widehat{\mathcal{C}}(x), \widehat{\mathcal{D}}=\widehat{\mathcal{D}}(x)$ and $\widehat{\mathcal{E}}=\widehat{\mathcal{E}}(x)$ are solutions of $(4.20)$

$$
\left[\begin{array}{ccccc}
\widehat{\alpha}_{n}(2 m+1) & 0 & 0 & 0 & 0 \\
\widehat{\beta}_{n}(2 m+1) & \widehat{\alpha}_{n}(2 m+2) & 0 & 0 & 0 \\
\widehat{\gamma}_{n}(2 m+1) & \widehat{\beta}_{n}(2 m+2) & \widehat{\alpha}_{n}(2 m+3) & 0 & 0 \\
\widehat{\delta}_{n}(2 m+1) & \widehat{\gamma}_{n}(2 m+2) & \widehat{\beta}_{n}(2 m+3) & \widehat{\alpha}_{n}(2 m+4) & 0 \\
\widehat{\varepsilon}_{n}(2 m+1) & \widehat{\delta}_{n}(2 m+2) & \widehat{\gamma}_{n}(2 m+3) & \widehat{\beta}_{n}(2 m+4) & \widehat{\alpha}_{n}(2 m+5)
\end{array}\right]\left[\begin{array}{c}
\widehat{\mathcal{A}}_{m} \\
\widehat{\mathcal{B}}_{m} \\
\widehat{\mathcal{C}}_{m} \\
\widehat{\mathcal{D}}_{m} \\
\widehat{\mathcal{E}}_{m}
\end{array}\right]=\left[\begin{array}{c}
\widehat{\mathcal{A}}_{m+1} \\
\widehat{\mathcal{B}}_{m+1} \\
\widehat{\mathcal{C}}_{m+1} \\
\widehat{\mathcal{D}}_{m+1} \\
\widehat{\mathcal{E}}_{m+1}
\end{array}\right],
$$

with initial conditions $\left(\widehat{\mathcal{A}}_{1}, \widehat{\mathcal{B}}_{1}, \widehat{\mathcal{C}}_{1}, \widehat{\mathcal{D}}_{1}, \widehat{\mathcal{E}}_{1}\right)=\left(\widehat{\alpha}_{n}, \widehat{\beta}_{n}, \widehat{\gamma}_{n}, \widehat{\delta}_{n}, \widehat{\varepsilon}_{n}\right)$. We will proceed by induction in $m$. For $m=1$ (4.19) holds by definition. Let $m \geq 1$ be such that (4.19) holds. Again recall that $d \equiv 1(\bmod p)$. Using that for $p \geq 7$ we have $2 d \geq 2(1+7)=16$ for all $n \geq 1$ we obtain

$$
\begin{aligned}
\widehat{\Delta}_{m+1}(\zeta) \equiv & \widehat{\Delta}_{m}(\widehat{h}(\zeta))-\widehat{\Delta}_{m}(\zeta) \\
\equiv & \widehat{\mathcal{A}}_{m}\left[\left(\zeta+\widehat{\Delta}_{1}\right)^{2 d m+1}-\zeta^{2 d m+1}\right]+\widehat{\mathcal{B}}_{m}\left[\left(\zeta+\widehat{\Delta}_{1}\right)^{2 d m+2}-\zeta^{2 d m+2}\right] \\
& +\widehat{\mathcal{C}}_{m}\left[\left(\zeta+\widehat{\Delta}_{1}\right)^{2 d m+3}-\zeta^{2 d m+3}\right]+\widehat{\mathcal{D}}_{m}\left[\left(\zeta+\widehat{\Delta}_{1}\right)^{2 d m+4}-\zeta^{2 d m+4}\right] \\
& +\widehat{\mathcal{E}}_{m}\left[\left(\zeta+\widehat{\Delta}_{1}\right)^{2 d m+5}-\zeta^{2 d m+5}\right] \\
\equiv & \widehat{\mathcal{A}}_{m}\left[\widehat{\alpha}_{n}(2 m+1) \zeta^{2 d m+3}+\widehat{\beta}_{n}(2 m+1) \zeta^{2 d m+4}+\widehat{\gamma}_{n}(2 m+1) \zeta^{2 d m+5}\right. \\
& \left.+\widehat{\delta}_{n}(2 m+1) \zeta^{2 d m+6}+\widehat{\varepsilon}_{n}(2 m+1) \zeta^{2 d m+7}\right] \\
& +\widehat{\mathcal{B}}_{m}\left[\widehat{\alpha}_{n}(2 m+2) \zeta^{2 d m+4}+\widehat{\beta}_{n}(2 m+2) \zeta^{2 d m+5}\right. \\
& \left.+\widehat{\gamma}_{n}(2 m+2) \zeta^{2 d m+6}+\widehat{\delta}_{n}(2 m+2) \zeta^{2 d m+7}\right] \\
& +\widehat{\mathcal{C}}_{m}\left[\widehat{\alpha}_{n}(2 m+3) \zeta^{2 d m+5}+\widehat{\beta}_{n}(2 m+3) \zeta^{2 d m+6}+\widehat{\gamma}_{n}(2 m+3) \zeta^{2 d m+7}\right] \\
& +\widehat{\mathcal{D}}_{m}\left[\widehat{\alpha}_{n}(2 m+4) \zeta^{2 d m+6}+\widehat{\beta}_{n}(2 m+4) \zeta^{2 d m+7}\right]+\widehat{\mathcal{E}}_{m}\left[\widehat{\alpha}_{n}(2 m+5) \zeta^{2 d m+7}\right] \\
\equiv & \widehat{\mathcal{A}}_{m+1} \zeta^{2 d m+3}+\widehat{\mathcal{B}}_{m+1} \zeta^{2 d m+4}+\widehat{\mathcal{C}}_{m+1} \zeta^{2 d m+5} \\
& +\widehat{\mathcal{D}}_{m+1} \zeta^{2 d m+6}+\widehat{\mathcal{E}}_{m+1} \zeta^{2 d m+7} \bmod \left\langle\zeta^{2 d m+8}\right\rangle .
\end{aligned}
$$

This completes the proof of the induction step and proves (4.19).

The first three equations in (4.20) were solved in [Nor17, page 267-268ff]. The solutions are

$$
\begin{gathered}
\widehat{\mathcal{A}}_{m}=\widehat{\alpha}_{n}^{m}(2 m-1) ! ! \\
\widehat{\mathcal{B}}_{m}=\widehat{\alpha}_{n}^{m-1} \widehat{\beta}_{n} \mathcal{R}_{m}, \\
\widehat{\mathcal{C}}_{m}=\widehat{\alpha}_{n}^{m-2} \widehat{\beta}_{n}^{2}\left(\mathcal{S}_{m}(2,0)-\mathcal{T}_{m}\right)+\widehat{\alpha}_{n}^{m-1} \widehat{\gamma}_{n} \mathcal{S}_{m}(0,1) .
\end{gathered}
$$

By substituting in $\widehat{\mathcal{D}}_{m}$ in(4.20) for the above equations we obtain

$$
\begin{aligned}
\widehat{\mathcal{D}}_{m+1}= & \widehat{\alpha}_{n}(2 m+4) \widehat{\mathcal{D}}_{m}+\widehat{\alpha}_{n}^{m} \widehat{\delta}_{n}(2 m+1) ! !+\widehat{\alpha}_{n}^{m-1} \widehat{\beta}_{n} \widehat{\gamma}_{n}(2 m+2) \mathcal{R}_{m} \\
& +\widehat{\alpha}_{n}^{m-2} \widehat{\beta}_{n}(2 m+3)\left(\widehat{\beta}_{n}^{2}\left(\mathcal{S}_{m}(2,0)-\mathcal{T}_{m}\right)+\widehat{\alpha}_{n} \widehat{\gamma}_{n} \mathcal{S}_{m}(0,1)\right),
\end{aligned}
$$

which, by Lemma 10 and Lemma 14 has the explicit solution

$$
\widehat{\mathcal{D}}_{m}=\widehat{\alpha}_{n}^{m-3}\left(\widehat{\alpha}_{n}^{2} \widehat{\delta}_{n} \mathcal{X}_{m}(0,1)+\widehat{\alpha}_{n} \widehat{\beta}_{n} \widehat{\gamma}_{n}\left(\mathcal{V}_{m}(0,2,2)+\mathcal{U}_{m}(0,1)\right)+\widehat{\beta}_{n}^{3}\left(\mathcal{U}_{m}(2,0)-\mathcal{W}_{m}\right)\right) .
$$


Insertion of all the above equations into the fifth equation in (4.20) yields

$$
\begin{aligned}
\widehat{\mathcal{E}}_{m+1}= & \widehat{\alpha}_{n}(2 m+5) \widehat{\mathcal{E}}_{m}+\widehat{\alpha}_{n}^{m-3}\left(\widehat{\alpha}_{n}^{3} \widehat{\varepsilon}_{n}(2 m+1) ! !+\widehat{\alpha}_{n}^{2} \widehat{\beta}_{n} \widehat{\delta}_{n}(2 m+2) \mathcal{R}_{m}\right. \\
& +\widehat{\alpha}_{n} \widehat{\beta}_{n}^{2} \widehat{\gamma}_{n}(2 m+3)\left(\mathcal{S}_{m}(2,0)-\mathcal{T}_{m}\right)+\widehat{\alpha}_{n}^{2} \widehat{\gamma}_{n}^{2}(2 m+3) \mathcal{S}_{m}(0,1) \\
& \left.+\widehat{\beta}_{n}(2 m+4)\left(\widehat{\alpha}_{n}^{2} \widehat{\delta}_{n} \mathcal{X}_{m}(0,1)+\widehat{\alpha}_{n} \widehat{\beta}_{n} \widehat{\gamma}_{n}\left(\mathcal{V}_{m}(0,2,2)+\mathcal{U}_{m}(0,1)\right)+\widehat{\beta}_{n}^{3}\left(\mathcal{U}_{m}(2,0)-\mathcal{W}_{m}\right)\right)\right)
\end{aligned}
$$

which, by Lemma 12, Lemma 13 and Lemma 14 has the explicit solution

$$
\begin{aligned}
\widehat{\mathcal{E}}_{m} & =\widehat{\alpha}_{n}^{m-4}\left(\widehat{\alpha}_{n}^{3} \widehat{\varepsilon}_{n} \mathcal{Z}_{m}(0,0,1)+\widehat{\alpha}_{n}^{2} \widehat{\beta}_{n} \widehat{\delta}_{n}\left(\widehat{\mathcal{R}}_{m}(0)+\widehat{\mathcal{X}}_{m}(0,1)\right)+\widehat{\beta}_{n}^{4}\left(\widehat{\mathcal{U}}_{m}(2,0)-\widehat{\mathcal{W}}_{m}\right)\right. \\
& \left.+\widehat{\alpha}_{n} \widehat{\beta}_{n}^{2} \widehat{\gamma}_{n}\left(\widehat{\mathcal{S}}_{m}(2,0,0)-\widehat{\mathcal{T}}_{m}(0)+\widehat{\mathcal{V}}_{m}(0,2,2)+\widehat{\mathcal{U}}_{m}(0,1)\right)+\widehat{\alpha}_{n}^{2} \widehat{\gamma}_{n}^{2} \widehat{\mathcal{S}}_{m}(0,1,0)\right)
\end{aligned}
$$

It follows from $(4.21)$ and $(4.22)$ and Lemma 4 that $\widetilde{\widehat{\mathcal{A}}}_{p}=\widetilde{\widehat{\mathcal{B}}}_{p}=0$. We also recall that

$$
\widehat{\alpha}_{n}=x_{1}^{p^{n}-2 d} \widehat{\varphi}, \quad \widehat{\beta}_{n}=\frac{x_{2}}{x_{1}} \widehat{\alpha}_{n}, \quad \widehat{\gamma}_{n}=-\left(\frac{3 x_{1}}{2}-\frac{x_{3}}{x_{1}}\right) \widehat{\alpha}_{n} .
$$

Concerning (4.23) together with (4.18) and by letting $m=p$ we obtain

$$
\begin{aligned}
\widetilde{\mathcal{C}}_{p} & =\widehat{\alpha}_{n}^{p-2} \widehat{\beta}_{n}^{2}\left(\widetilde{\mathcal{S}}_{p}(2,0)-\widetilde{\mathcal{T}}_{p}\right)+\widehat{\alpha}_{n}^{p-1} \widehat{\gamma}_{n} \widetilde{\mathcal{S}}_{p}(0,1) \\
& =\widehat{\alpha}_{n}^{p-2}\left(\widehat{\beta}_{n}^{2}-\widehat{\alpha}_{n} \widehat{\gamma}_{n}\right) \\
& =\widehat{\alpha}_{n}^{p} x_{1}^{-2} \widehat{\varphi}=\left(x_{1}^{p^{n}-2 d} \widehat{\varphi}^{d}\right)^{p} x_{1}^{-2} \widehat{\varphi} \\
& =x_{1}^{p^{n+1}-2(d p+1)} \widehat{\varphi}^{d p+1}=\widehat{\alpha}_{n+1} .
\end{aligned}
$$

Similarly for (4.24) we obtain

$$
\begin{aligned}
\widetilde{\mathcal{D}}_{p} & =\widehat{\alpha}_{n}^{p-3}\left(\widehat{\alpha}_{n}^{2} \widehat{\delta}_{n} \widetilde{\mathcal{X}}_{p}(0,1)+\widehat{\alpha}_{n} \widehat{\beta}_{n} \widehat{\gamma}_{n}\left(\widetilde{\mathcal{V}}_{p}(0,2,2)+\widetilde{\mathcal{U}}_{p}(0,1)\right)+\widehat{\beta}_{n}^{2}\left(\widetilde{\mathcal{U}}_{p}(2,0)-\widetilde{\mathcal{W}}_{p}\right)\right) \\
& =\widehat{\alpha}_{n}^{p-3}\left(\widehat{\beta}_{n}^{3}-\widehat{\alpha}_{n} \widehat{\beta}_{n} \widehat{\gamma}_{n}\right) .
\end{aligned}
$$

By using $\widehat{\alpha}_{n}^{p-2}\left(\widehat{\beta}_{n}^{2}-\widehat{\alpha}_{n} \widehat{\gamma}_{n}\right)=\widehat{\alpha}_{n+1}$ from (4.26) we obtain

$$
\widetilde{\widehat{\mathcal{D}}}_{p}=\frac{x_{2}}{x_{1}} \widehat{\alpha}_{n+1}=\widehat{\beta}_{n+1} \text {. }
$$

Finally for (4.25) again by using $\widehat{\alpha}_{n}^{p-2}\left(\widehat{\beta}_{n}^{2}-\widehat{\alpha}_{n} \widehat{\gamma}_{n}\right)=\widehat{\alpha}_{n+1}$ together with Lemma 12 and 13

$$
\begin{aligned}
\widetilde{\widehat{\mathcal{E}}}_{p}= & \widehat{\alpha}_{n}^{p-4}\left(\widehat{\alpha}_{n}^{3} \widehat{\varepsilon}_{n} \widetilde{\mathcal{Z}}_{p}(0,0,1)+\widehat{\alpha}_{n}^{2} \widehat{\beta}_{n} \widehat{\delta}_{n}\left(\widetilde{\widehat{\mathcal{R}}}_{p}(0)+\widetilde{\widehat{\mathcal{X}}}_{p}(0,1)\right)+\widehat{\beta}_{n}^{4}\left(\widetilde{\widehat{\mathcal{U}}}_{p}(2,0)-\widetilde{\widehat{\mathcal{W}}}_{p}\right)\right. \\
& \left.+\widehat{\alpha}_{n} \widehat{\beta}_{n}^{2} \widehat{\gamma}_{n}\left(\widetilde{\mathcal{\mathcal { S }}}_{p}(2,0,0)-\widetilde{\widehat{\mathcal{T}}}_{p}(0)+\widetilde{\widehat{\mathcal{V}}}_{p}(0,2,2)+\widetilde{\widehat{\mathcal{U}}}_{p}(0,1)\right)+\widehat{\alpha}_{n}^{2} \widehat{\gamma}_{n}^{2} \widetilde{\mathcal{\mathcal { S }}}_{p}(0,1,0)\right) \\
= & \widehat{\alpha}_{n}^{p-4}\left(\widehat{\alpha}_{n} \widehat{\beta}_{n}^{2} \widehat{\gamma}_{n}-\widehat{\alpha}_{n}^{2} \widehat{\gamma}_{n}^{2}\right) \\
= & -\left(\frac{3 x_{1}}{2}-\frac{x_{3}}{x_{1}}\right) \widehat{\alpha}_{n+1}=\widehat{\gamma}_{n+1} .
\end{aligned}
$$

Thus, the proof is finished by specializing for each $i \in[1,3] x_{i}$ to $a_{i+1}$ which yields

$$
\widetilde{\Delta}_{p}(\zeta) \equiv \alpha_{n+1} \zeta^{2(d p+1)+1}+\beta_{n+1} \zeta^{2(d p+1)+2}+\gamma_{n+1} \zeta^{2(d p+1)+3} \bmod \left\langle\zeta^{2(d p+1)+4}\right\rangle .
$$

This completes the proof of Theorem B. 


\section{Appendix A. Details for Remark 2}

Let $p \geq 5$ and let $h \in k[[\zeta]]$ of the form $h(\zeta) \equiv \zeta\left(1+x_{3} \zeta^{3}\right) \bmod \left\langle\zeta^{10}\right\rangle$, then by putting $q:=3$, $a_{1}:=\frac{x_{3}}{3}$ and $a_{2}:=-\frac{4}{9} a_{3}^{2}$ in (3.2) in [LRL16a, Main Lemma] we have $i_{n}(h)=3\left(1+p+\cdots+p^{n}\right)$.

We recall that $0<\left|a_{2}\right|<1$ and $\left|a_{3}\right|=1$, thus we have $\widetilde{f}(\zeta)=\zeta\left(1+a_{3} \zeta^{3}\right) \bmod \left\langle\zeta^{10}\right\rangle$, and by the previous argument we have $\widetilde{f}$ is 3 -ramified as required.

\section{Appendix B. Details for Example 1}

Let $q_{1}(\zeta)=\zeta+(1+t) \zeta^{3}+\zeta^{4}$, and $q_{2}(\zeta)=\zeta+(2+t) \zeta^{3}+4 \zeta^{4}+4 \zeta^{5}$. In both cases $q_{1}$ and $q_{2}$ we have $\lambda \neq 0$. Thus, both $q_{1}$ and $q_{2}$ are 2-ramified. However, for the reduction we first note that $i_{0}\left(\widetilde{q_{1}}\right)=i_{0}\left(\widetilde{q_{2}}\right)=2$. It follows that neither $\widetilde{q_{1}}$ nor $\widetilde{q_{2}}$ is 3-ramified. However, note that

$$
\frac{3}{2} 1^{3}+1^{2} \equiv 0 \quad(\bmod 5), \quad \text { and } \quad \frac{3}{2} 2^{3}+4^{2}-2 \cdot 4 \equiv 0 \quad(\bmod 5),
$$

so neither $\widetilde{q_{1}}$ nor $\widetilde{q_{2}}$ is 2-ramified. In fact $i_{1}\left(\widetilde{q}_{1}\right)=17$ and $i_{1}\left(\widetilde{q}_{2}\right)=27$. By [LS98, Corollary 1]

$$
i_{n}\left(\widetilde{q_{1}}\right)=2+3 p+\cdots+3 p^{n}, \quad \text { and } \quad i_{n}\left(\widetilde{q_{2}}\right)=2+5 p+\cdots+5 p^{n} .
$$

Thus, $i_{n}\left(\widetilde{q_{1}}\right)-i_{n-1}\left(\widetilde{q_{1}}\right)=3 p^{n}$, and by Lemma 1 the norm of the periodic points in $\mathbb{F}_{5}((t))$ of minimal period $p^{n}$, with $n \geq 1$, are in the case of $q_{1}$ equal to $|\lambda|^{\frac{1}{p}}$. For $q_{2}$ we have $i_{n}\left(\widetilde{q_{2}}\right)-$ $i_{n-1}\left(\widetilde{q_{2}}\right)=5 p^{n}$. The periodic points of $q_{2}$, that are not fixed points, are thus in $\left\{\zeta \in \mathbb{F}_{5}((t))\right.$ : $\left.|\zeta|>|\lambda|^{\frac{1}{p}}\right\}$.

\section{REFERENCES}

[AK09] Vladimir Anashin and Andrei Khrennikov. Applied Algebraic Dynamics. Walter de Gruyter, Berlin, 2009.

[AV94] David Arrowsmith and Franco Vivaldi. Geometry of $p$-adic Siegel discs. Physica D, 71:222-236, 1994.

[Ela05] Saber Elaydi. An introduction to difference equations. Springer, New York, 3. ed. edition, 2005.

[Lan02] Serge Lang. Algebra. Springer, New York, rev. 3. ed. edition, 2002.

[Lin13] Karl-Olof Lindahl. The size of quadratic p-adic linearization disks. Adv. Math., 248:872-894, 2013.

[LRL16a] Karl-Olof Lindahl and Juan Rivera-Letelier. Generic parabolic points are isolated in positive characteristic. Nonlinearity, 29(5):1596-1621, 2016.

[LRL16b] Karl-Olof Lindahl and Juan Rivera-Letelier. Optimal cycles in ultrametric dynamics and minimally ramified power series. Compos. Math., 152(1):187-222, 2016.

[LS98] François Laubie and M. Saïne. Ramification of Some Automorphisms of Local Fields. Journal of Number Theory, 72(2):174-182, 1998.

[Lub94] Jonathan Lubin. Non-Archimedean dynamical systems. Compo. Math., 94(3):321-346, 1994.

[Lub95] Jonathan Lubin. Sen's theorem on iteration of power series. Proceedings of the American Mathematical Society, 123(1):63-66, 1995.

[MA15] Farrukh Mukhamedov and Hasan Akin. On non-Archimedean recurrence equations and their applications. J. Mathematical Analysis and Applications, 423(2):1203-1218, 2015.

[Nor17] Jonas Nordqvist. Characterization of 2-ramified power series. J. Number Theory, 174:258-273, 2017.

[RL03] Juan Rivera-Letelier. Dynamique des functionsrationelles sur des corps locaux, Astérisque, 287:147230, 2003.

[Sen69] Shankar Sen. On automorphisms of local fields. Ann. of Math. (2), 90:33-46, 1969.

[Sil07] Joseph Silverman. The Arithmetic of Dynamical Systems. Graduate Texts in Mathematics, 241. Springer, New York, 2007. 